TRANSACTIONS OF THE

AMERICAN MATHEMATICAL SOCIETY

Volume 352, Number 4, Pages 1859-1888

S 0002-9947(99)02425-3

Article electronically published on October 29, 1999

\title{
A BANACH SPACE BLOCK FINITELY UNIVERSAL FOR MONOTONE BASES
}

\author{
E. ODELL AND TH. SCHLUMPRECHT
}

\begin{abstract}
A reflexive Banach space $X$ with a basis $\left(e_{i}\right)$ is constructed having the property that every monotone basis is block finitely representable in each block basis of $X$.
\end{abstract}

\section{INTRODUCTION}

B. Maurey and H. Rosenthal $[\mathrm{MR}$ in 1977 gave an example of a normalized weakly null sequence for which the summing basis is block finitely represented in all subsequences. They asked whether one could for some basis achieve this in all block bases and thereby solve in the negative the unconditional basic sequence problem. The later problem was subsequentially solved in 1991 in a spectacular paper of W.T. Gowers and B. Maurey [GM]. Further examples have since been given solving a myriad of related problems. These examples all have a basis $\left(e_{i}\right)$ with the property that for some sequence $c_{n} \nearrow \infty$ if $\left(y_{i}\right)$ is any block basis of $\left(e_{i}\right)$, then for all $n \in \mathbb{N}$ and $\varepsilon>0$ there is a block basis $\left(z_{i}\right)_{i=1}^{n}$ of $\left(y_{i}\right)$ with

$$
\frac{\left\|\sum_{i=1}^{n} z_{i}\right\|}{\left\|\sum_{i=1}^{n}(-1)^{i} z_{i}\right\|}>c_{n}-\varepsilon .
$$

In known examples $c_{n}$ grows rather slowly (e.g., like $\log n$ ). In this paper we answer in the affirmative the problem of B. Maurey and H. Rosenthal. Thus we obtain an example for which one has $c_{n}=n$, the worst possible unconditionality.

Our example is a "conditional version" of an example in [OS] in which an unconditional basis is constructed so that all finite 1-unconditional bases are block finitely represented in all block bases. The norm in that example satisfies the implicit equation

$$
\|x\|=\max \left\{\|x\|_{\infty}, \sup \left\{\frac{1}{f(\ell)} \sum_{j=1}^{\ell}\left\|E_{i}(x)\right\|_{m_{i}}: \begin{array}{c}
\ell \in \mathbb{N}, m_{i} \in \mathbb{N}, \text { for } i=1, \ldots, \ell \\
\left(m_{i}, E_{i}\right)_{i=1}^{\ell} \text { is admissible }
\end{array}\right\}\right\},
$$

where $\|x\|_{m} \equiv \sup \left\{\frac{1}{m} \sum_{i=1}^{m}\left\|F_{i}(x)\right\|: F_{1}<\cdots<F_{m}\right\}$. In (1.1) $f(\ell) \equiv \log _{2}(\ell+1)$ and $\left(m_{i}, E_{i}\right)_{i=1}^{\ell}$ is admissible if $m_{1}>2, f\left(m_{i+1}\right)>\sum_{j=1}^{i}\left|E_{j}\right|$, and $E_{1}<E_{2}<$ $\cdots<E_{\ell}$ (the notation will be described in $\S 2$ below).

Received by the editors May 24, 1996.

1991 Mathematics Subject Classification. Primary 46B20; Secondary 46B15, 46B03.

Key words and phrases. Unconditional basic sequence, block finitely universal.

Research supported by NSF and TARP. 
The idea of "defining" a norm by an implicit equation is due to T. Figiel and W.B. Johnson FJ in their paper on Tsirelson's space. We might describe the norm in (1.1) as a "2-layer" Tsirelson type norm. The example in this paper is a "3-layer" norm. Also, terms like " $\left\|F_{i}(x)\right\|$ " need to be replaced by " $x_{i}^{*}(x)$ " where the $x_{i}$ 's are rather carefully chosen in the manner of [GM]. It is also possible to define the norms in (1.1) and below by describing the dual ball much in the same manner as Tsirelson did in his original paper $[\mathrm{T}]$.

\section{Terminology AND USEFul FACTS}

The linear space of all finitely supported real valued functions on $\mathbb{N}$ is denoted by $c_{00}$. For nonempty $E, F \subseteq \mathbb{N}$, " $E<F$ " means that $\max E<\min F$. $|E|$ is the cardinality of $E$. For a sequence of nonzero elements $\left(x_{i}\right) \subseteq c_{00}$, " $x_{1}<x_{2}<\ldots$ " means that $\operatorname{supp} x_{i}<\operatorname{supp} x_{2}<\cdots$ where $\operatorname{supp} x_{i}=\left\{j \in \mathbb{N}: x_{i}(j) \neq 0\right\}$. If $x \in c_{00}$ and $E \subseteq \mathbb{N}$, then $E x \in c_{00}$ is defined by $E x(j)=x(j)$ if $j \in E$ and 0 otherwise. $\left(e_{i}\right)_{1}^{\infty}$ is the unit vector basis for $c_{00} .\left(e_{i}^{*}\right)_{1}^{\infty}$ are the biorthogonal functionals to $\left(e_{i}\right)$.

We shall be interested in a collection $\mathcal{N}$ of certain type norms on $c_{00} \cdot\|\cdot\| \in \mathcal{N}$ if $\left(e_{i}\right)$ is a normalized monotone basis for the completion of $\left(c_{00},\|\cdot\|\right)$ and $\left\|e_{i}^{*}\right\|=1$ for all $i$. The latter just says that $\left\|\sum a_{i} e_{i}\right\| \geq \max _{i}\left|a_{i}\right| \equiv\left\|\left(a_{i}\right)\right\|_{\infty}$ for all $\left(a_{i}\right) \in c_{00}$. All $\ell_{p}$ norms and the Tsirelson norm are in $\mathcal{N}$. There is a natural partial order on $\mathcal{N}:\|\cdot\| \leq|\cdot|$ if $\|x\| \leq|x|$ for all $x \in c_{00}$. Clearly, $\|\cdot\|_{c_{0}} \leq|\cdot| \leq\|\cdot\|_{\ell_{1}}$ if $|\cdot| \in \mathcal{N}$.

If $\|\cdot\| \in \mathcal{N}$ and $X=\left(c_{00},\|\cdot\|\right)$, then $B\left(X^{*}\right)$, the unit ball of $X^{*}$, is the weak* closure of $\left\{\sum_{1}^{n} a_{i} e_{i}^{*}: n \in \mathbb{N},\left(a_{i}\right)_{1}^{n} \subseteq \mathbb{R}\right\} \cap B\left(X^{*}\right)$ and as such may be identified with

$$
\overline{\left\{\left(a_{i}\right) \in c_{00}:\left\|\sum a_{i} e_{i}^{*}\right\| \leq 1\right\}} \subseteq[-1,1]^{\mathbb{N}}
$$

where $[-1,1]^{\mathbb{N}}$ is given the product topology.

There are a number of Tsirelson type spaces in the literature described by implicit equations much like (1.1) ([CS, [AD, GM], OS] $)$. These implicit norms are fixed points of certain mappings on $\mathcal{N}$. The next proposition gives a general argument for their existence.

Proposition 2.1. Let $P: \mathcal{N} \rightarrow \mathcal{N}$ be order preserving $(|\cdot| \leq\|\cdot\| \Rightarrow P|\cdot| \leq P\|\cdot\|)$. Then $P$ admits a smallest fixed point. Thus there exists $\|\cdot\| \in \mathcal{N}$ with

a) $\|\cdot\|=P\|\cdot\|$.

b) If $P|\cdot|=|\cdot|$, then $\|\cdot\| \leq|\cdot|$.

Proof. Let $\|\cdot\|_{0} \equiv\|\cdot\|_{\infty}$. By transfinite induction we define $\|\cdot\|_{\alpha}$ for $\alpha<\omega_{1}$. If $\alpha=\beta+1$, we set

$$
\|x\|_{\alpha}=\max \left(\|x\|_{\beta}, P\|x\|_{\beta}\right) \text {. }
$$

If $\alpha$ is a limit ordinal, we set $\|x\|_{\alpha}=\sup _{\beta<\alpha}\|x\|_{\beta}$. Clearly $\|\cdot\|_{\alpha} \in \mathcal{N}$ for all $\alpha<\omega_{1}$. Since $\left(\|\cdot\|_{\alpha}\right)_{\alpha<\omega_{1}}$ is an increasing family of norms the dual balls $B_{\alpha}^{*} \equiv B\left(c_{00},\|\cdot\|_{\alpha}^{*}\right)$ are increasing closed subsets of $[-1,1]^{\mathbb{N}}$. Since this space is compact metrizable there exists $\alpha_{0}<\omega_{1}$ so that $B_{\alpha_{0}}^{*}=B_{\beta}^{*}$ for all $\beta \geq \alpha_{0}$. Thus $\|\cdot\|_{\alpha_{0}}=\|\cdot\|_{\beta}$ for $\beta \geq \alpha_{0}$ as well. We set $\|\cdot\|=\|\cdot\|_{\alpha_{0}}$.

To see that $\|\cdot\|$ is a fixed point for $P$ we first note that $\|\cdot\|=\|\cdot\|_{\alpha_{0}+1} \geq$ $P\|\cdot\|_{\alpha_{0}}=P\|\cdot\|$. For the reverse inequality it suffices to show by induction that $\|\cdot\|_{\alpha} \leq P\|\cdot\|$ for all $\alpha<\omega_{1}$. Clearly this holds for $\alpha=0$ and if $\|\cdot\|_{\beta} \leq P\|\cdot\|$, 
then $\|\cdot\|_{\beta+1}=\max \left(\|\cdot\|_{\beta}, P\|\cdot\|_{\beta}\right) \leq P\|\cdot\|$. Indeed $\|\cdot\|_{\beta} \leq\|\cdot\|$ by the definition of $\|\cdot\|$ and so $P\|\cdot\|_{\beta} \leq P\|\cdot\|$ since $P$ is order preserving. Also if $\alpha$ is a limit ordinal and $\|\cdot\|_{\beta} \leq P\|\cdot\|$ for $\beta<\alpha$, then $\|\cdot\|_{\alpha} \leq P\|\cdot\|$. Thus $P\|\cdot\|=\|\cdot\|$.

To see b), let $P|\cdot|=|\cdot|$. Then $\|\cdot\|_{0} \leq|\cdot|$ and by induction we easily obtain $\|\cdot\|_{\alpha} \leq|\cdot|$ for $\alpha<\omega_{1}$, hence $\|\cdot\| \leq|\cdot|$.

Basic sequences $\left(x_{i}\right)$ and $\left(y_{i}\right)$ are $C$-equivalent if for some constants $\alpha, \beta$ with $\alpha^{-1} \beta \leq C, \alpha\left\|\sum a_{i} x_{i}\right\| \leq\left\|\sum a_{i} y_{i}\right\| \leq \beta\left\|\sum a_{i} x_{i}\right\|$ for all $\left(a_{i}\right) \in c_{00}$. A basic sequence $\left(z_{i}\right)$ is block finitely represented in a basic sequence $\left(x_{i}\right)$ if for all $\varepsilon>0$ and $n \in \mathbb{N}$ there exists a block basis $\left(y_{i}\right)_{1}^{n}$ of $\left(x_{i}\right)$ which is $(1+\varepsilon)$-equivalent to $\left(z_{i}\right)_{1}^{n}$.

Let $\|\cdot\| \in \mathcal{N}$ and $X=\left(c_{00},\|\cdot\|\right)$. For $A \subseteq X^{*}$ and $x \in X$ we set $\|x\|_{A}=$ $\sup \left\{\left|x^{*}(x)\right|: x^{*} \in A\right\}$. For $1 \leq p \leq \infty, C \geq 1$ and $k \in \mathbb{N}, x \in X$ is called an $\ell_{p}^{k}$-average with constant $C$ if $x=k^{-1 / p} \sum_{i=1}^{k} x_{i}$ for some normalized sequence $x_{1}<\cdots<x_{k}$ which is $C$-equivalent to the unit vector basis of $\ell_{p}^{k}$.

If $\|\cdot\| \in \mathcal{N}$ and $X=\left(c_{00},\|\cdot\|\right)$, then $\|\cdot\|^{*} \in \mathcal{N}$ as well. Indeed $\left(e_{i}^{*}\right)$ is a normalized monotone basic sequence in $X^{*}$ and $\left\|\sum a_{i} e_{i}^{*}\right\| \geq \max \left|a_{i}\right|$ for $\left(a_{i}\right) \in c_{00}$. So we are free to use our notation $x^{*}<y^{*}$ for elements of the dual in $\operatorname{span}\left(e_{i}^{*}\right)$ as well.

Before defining our norm we present some technical notation and a lemma. The lemma could be postponed but it does help one become familiar with the terminology. Our first definitions are motivated by [GM].

Fix $H \subset c_{00} \cap[-1,1]^{\mathbb{N}}$, a countable subset of nonzero elements. Let $\mathcal{D}=\left(D_{i}\right)$ be a sequence of subsets of $H$. Let

$$
\sigma:\left\{\left(x_{1}^{*}, \ldots, x_{n}^{*}\right): n \in \mathbb{N}, x_{i}^{*} \in H \text { for } i \leq n, x_{1}^{*}<\cdots<x_{n}^{*}\right\} \rightarrow \mathbb{N}
$$

be an injective map satisfying the following condition:

$$
\sigma\left(x_{1}^{*}, \ldots, x_{n}^{*}\right)>\max \left(k, \max \operatorname{supp} x_{n}^{*}\right) \text { if } x_{n}^{*} \in D_{k} .
$$

Let $M=\left(M_{n}\right)$ be a subsequence of $\mathbb{N}$.

Definition. $\left(x_{1}^{*}, \ldots, x_{n}^{*}\right) \subseteq c_{00}$ is $(\mathcal{D}, M, \sigma)$-admissible if

1) $x_{1}^{*}<\cdots<x_{n}^{*}$

2) $x_{1}^{*} \in \bigcup_{i>M_{n}} D_{i}$,

3) $x_{i+1}^{*} \in \bar{D}_{\sigma\left(x_{1}^{*}, \ldots, x_{i}^{*}\right)}$ if $1 \leq i<n$.

We have used " $x_{i}^{*}$ " above in our definitions because we will be applying this for elements in $X^{*}$.

Let $f:[1, \infty) \rightarrow \mathbb{R}$ be given by $f(x)=\log _{2}(x+1)$. We will make use of the fact that $f$ is strictly increasing, $f(1)=1$ and both $f(x)$ and $\frac{x}{f(x)}$ are concave functions.

For $n \in \mathbb{N}$ set

$$
\Gamma_{n}=\Gamma_{n}(\mathcal{D}, M, \sigma)=\left\{\frac{1}{f(n)} \sum_{j=1}^{n}:\left(x_{j}^{*}\right)_{1}^{n} \text { is }(\mathcal{D}, M, \sigma) \text {-admissible }\right\} .
$$

Let $\Gamma=\Gamma(\mathcal{D}, M, \sigma)=\bigcup_{\mathbb{N}} \Gamma_{n}, D=\bigcup_{i \geq M_{1}} D_{i}$ and note that $\Gamma_{1}=D$.

Lemma 2.2. Let $\|\cdot\| \in \mathcal{N}, X=\left(c_{00},\|\cdot\|\right)$ and suppose that $\Gamma(\mathcal{D}, M, \sigma) \subseteq B\left(X^{*}\right)$.

a) Let $k, m \in \mathbb{N}, \varepsilon>0$ and let $e_{k}<y_{1}<\cdots<y_{m}$ be a block sequence of nonzero elements in $B(X) \cap[-1,1]^{\mathbb{N}}$. Assume that for all $1<i \leq m$ and for any 
$(\mathcal{D}, M, \sigma)$-admissible sequence $\left(x_{1}^{*}, \ldots, x_{j}^{*}\right)$ with $\max \operatorname{supp} x_{j}^{*} \geq \min \operatorname{supp} y_{i}$ we have for all $x^{*} \in \bigcup_{i \geq \sigma\left(x_{1}^{*}, \ldots, x_{j}^{*}\right)} D_{i}$,

$$
\left|x^{*}\left(y_{i}\right)\right| \leq \frac{\varepsilon}{f^{-1}\left(\frac{1}{\varepsilon} \max \operatorname{supp} y_{i-1}\right)} .
$$

Let $\ell \in \mathbb{N}, x^{*}=\frac{1}{f(\ell)} \sum_{j=1}^{\ell} x_{j}^{*} \in \Gamma_{\ell}$ and $y=\sum_{i=1}^{m} \alpha_{i} y_{i}$ with $\left(\alpha_{i}\right)_{1}^{m} \subseteq \mathbb{R}$ so that $x^{*}(y) \neq 0$. Set

$$
j_{1} \equiv \min \left\{j \leq \ell: \max \operatorname{supp} x_{j}^{*} \geq \min \operatorname{supp} y\right\}
$$

Then

$$
\begin{aligned}
\left|x^{*}(y)\right| \leq & \frac{1}{f(\ell)}\left|x_{j_{1}}^{*}(y)\right|+\max _{i \leq m}\left|\alpha_{i}\right|\left|\left(x^{*}-\frac{x_{j_{1}}^{*}}{f(\ell)}\right) y_{i}\right| \\
& +\frac{\min (m-1, \ell-1)}{f(\ell)} \sup \left\{\left|x^{*}(y)\right|: x^{*} \in \bigcup_{t \geq k} D_{t}\right\} \\
& +\frac{\min (2, \ell-1)}{f(\ell)} \sup \left\{\left|x^{*}\left(\alpha_{i} y_{i}\right)\right|: i \leq m, x^{*} \in \bigcup_{t \geq k} D_{t}\right\}+2 \varepsilon\left\|\left(\alpha_{i}\right)\right\|_{\infty} \\
\leq & \frac{1}{f(\ell)}\left|x_{j_{1}}^{*}(y)\right|+\frac{\min (m-1, \ell-1)}{f(\ell)} \sup \left\{\left|x^{*}(y)\right|: x^{*} \in \bigcup_{t \geq k} D_{t}\right\} \\
& +\left\|\left(\alpha_{i}\right)\right\|_{\infty}\left[\min (\ell-1,1)\left(1+\frac{1}{f(\ell)}\right)+2 \varepsilon\right. \\
& \left.+\sup \left\{\left|x^{*}\left(y_{i}\right)\right|: i \leq m, x^{*} \in \bigcup_{t \geq k} D_{t}\right\}\right] .
\end{aligned}
$$

b) Let $\left(y_{i}\right)_{i=1}^{\infty} \subseteq B(X) \cap[-1,1]^{\mathbb{N}}$ be a block basis of $\left(e_{i}\right)$ satisfying

$$
\varepsilon_{n} \equiv \sup \left\{\left|x^{*}\left(y_{i}\right)\right|: i \geq n, x^{*} \in \bigcup_{t \geq n} D_{t}\right\} \rightarrow 0
$$

as $n \rightarrow \infty$.

Then for all $\ell, m \in \mathbb{N}$ and $\left(\alpha_{i}\right)_{1}^{m} \subseteq \mathbb{R}$ we have

$$
\varlimsup_{n_{1} \rightarrow \infty} \ldots \varlimsup_{n_{m} \rightarrow \infty}\left\|\sum_{i=1}^{m} \alpha_{i} y_{n_{i}}\right\|_{\Gamma_{\ell}} \leq \frac{1}{f(\ell)} \varlimsup_{n_{1} \rightarrow \infty} \ldots \varlimsup_{n_{m} \rightarrow \infty}\left\|\sum_{i=1}^{m} \alpha_{i} y_{n_{i}}\right\|_{D}
$$

and

$$
\varlimsup_{n_{1} \rightarrow \infty} \ldots \varlimsup_{n_{m} \rightarrow \infty}\left\|\sum_{i=1}^{m} \alpha_{i} y_{n_{i}}\right\|_{\Gamma} \leq \max \left\{\left\|\left(\alpha_{i}\right)\right\|_{\infty}, \varlimsup_{n_{1} \rightarrow \infty} \ldots \varlimsup_{n_{m} \rightarrow \infty}\left\|\sum_{i=1}^{m} \alpha_{i} y_{n_{i}}\right\|_{D}\right\} .
$$

Proof. a) We begin by choosing for $1 \leq i \leq m, \tilde{I}_{i} \subseteq\left\{j_{1}+1, \ldots, \ell\right\}$ to be those $j$ 's for which $x_{j}^{*}$ acts only on $y_{i}$. For $1<i<m$

$$
\tilde{I}_{i}=\left\{j>j_{1}: \operatorname{supp} x_{j}^{*} \cap \operatorname{supp} y_{i} \neq \emptyset \text { and } y_{i-1}<x_{j}^{*}<y_{i+1}\right\} .
$$


We take

$$
\begin{aligned}
\tilde{I}_{1} & =\left\{j>j_{1}: \operatorname{supp} x_{j}^{*} \cap \operatorname{supp} y_{1} \neq \emptyset \text { and } x_{j}^{*}<y_{2}\right\}, \\
\tilde{I}_{m} & =\left\{j>j_{1}: \operatorname{supp} x_{j}^{*} \cap \operatorname{supp} y_{m} \neq \emptyset \text { and } y_{m-1}<x_{j}^{*}\right\}
\end{aligned}
$$

For $2 \leq i \leq m$ it may happen that there exists (at most one) $j_{i}>j_{1}$ with min $\operatorname{supp} x_{j_{i}}^{*} \leq \max \operatorname{supp} y_{i-1}$ and min supp $y_{i} \leq \max \operatorname{supp} x_{j_{i}}^{*}$ and if $i<m$, $\max \operatorname{supp} x_{j_{i}}^{*}<\min \operatorname{supp} y_{i+1}$. In this case we take $I_{i}=\tilde{I}_{i}$. Otherwise, if $\tilde{I}_{i} \neq \emptyset$, we set $j_{i}=\min \tilde{I}_{i}$ and $I_{i}=\tilde{I}_{i} \backslash\left\{j_{i}\right\}$. Let $I_{0}$ be the set of all $j_{i}$ 's, $i \geq 2$, thus obtained and note that $\left|I_{0}\right| \leq \min (\ell-1, m-1)$. Let $I_{1}=\tilde{I}_{1}$. We thus have

$$
\left\{j_{1}\right\} \cup \bigcup_{i=0}^{m} I_{i} \supseteq\left\{j \leq \ell: x_{j}^{*}(y) \neq 0\right\} .
$$

Finally we set

$$
i_{0}=\min \left\{i \leq m: f(\ell) \leq \frac{\operatorname{maxsupp} y_{i}}{\varepsilon}\right\}
$$

and $i_{0}=m$ if the set is empty. Since $y, x^{*} \in[-1,1]^{\mathbb{N}}$,

$$
\sum_{i=1}^{i_{0}-1} \frac{1}{f(\ell)} \sum_{j \in I_{i}}\left|x_{j}^{*}(y)\right| \leq \frac{\max \operatorname{supp} y_{i_{0}-1}}{f(\ell)}<\varepsilon .
$$

For $m \geq i>i_{0}$ and $j \in I_{i}$ it follows from $(2.2 .1)$ and the fact that $\left(x_{1}^{*}, \ldots, x_{j-1}^{*}\right)$ is $(\mathcal{D}, M, \sigma)$-admissible with $\max \operatorname{supp} x_{j-1}^{*} \geq \min \operatorname{supp} y_{i}$ that

$$
\left|x_{j}^{*}\left(y_{i}\right)\right|<\frac{\varepsilon}{f^{-1}\left(\frac{\max \operatorname{supp} y_{i-1}}{\varepsilon}\right)} \leq \frac{\varepsilon}{f^{-1}\left(\frac{\operatorname{max\operatorname {supp}y} y_{0}}{\varepsilon}\right)}<\frac{\varepsilon}{\ell}
$$

where the last inequality uses the definition of $i_{0}$. Thus

$$
\sum_{i=i_{0}+1}^{m} \frac{1}{f(\ell)} \sum_{j \in I_{i}}\left|x_{j}^{*}\left(y_{i}\right)\right|<\sum_{i=i_{0}+1}^{m} \frac{1}{f(\ell)}\left|I_{i}\right| \frac{\varepsilon}{\ell} \leq \varepsilon .
$$

We are now ready to estimate $\left|x^{*}(y)\right|$.

$$
\begin{aligned}
\left|x^{*}(y)\right|=\mid \frac{x_{j_{1}}^{*}(y)}{f(\ell)}+\alpha_{i_{0}}\left(x^{*}-\frac{x_{j_{1}}^{*}}{f(\ell)}\right)\left(y_{i_{0}}\right) & +\frac{1}{f(\ell)} \sum_{j \in I_{0}} x_{j}^{*}\left(y-\alpha_{i_{0}} y_{i_{0}}\right) \\
& +\frac{1}{f(\ell)} \sum_{i \neq i_{0}} \sum_{j \in I_{i}} x_{j}^{*}\left(\alpha_{i} y_{i}\right) \mid \\
\leq & \frac{1}{f(\ell)}\left|x_{j_{1}}^{*}(y)\right|+\left|\alpha_{i_{0}}\right|\left|\left(x^{*}-\frac{x_{j_{1}}^{*}}{f(\ell)}\right)\left(y_{i_{0}}\right)\right|+\frac{1}{f(\ell)} \sum_{j \in I_{0}}\left|x_{j}^{*}(y)\right| \\
& +\frac{1}{f(\ell)} \sum_{j \in I_{0}}\left|\alpha_{i_{0}}\right|\left|x_{j}^{*}\left(y_{i_{0}}\right)\right|+2 \varepsilon\left\|\left(\alpha_{i}\right)\right\|_{\infty},
\end{aligned}
$$


where the very last estimate follows from (1) and (2). Thus

$$
\begin{aligned}
\left|x^{*}(y)\right| \leq & \frac{1}{f(\ell)}\left|x_{j_{1}}^{*}(y)\right|+\max _{i \leq m}\left|\alpha_{i}\right|\left|\left(x^{*}-\frac{x_{j_{1}}^{*}}{f(\ell)}\right)\left(y_{i}\right)\right| \\
& +\frac{\min (m-1, \ell-1)}{f(\ell)} \sup \left\{\left|x^{*}(y)\right|: x^{*} \in \bigcup_{t \geq k} D_{t}\right\} \\
& +\frac{\min (2, \ell-1)}{f(\ell)} \sup \left\{\left|x^{*}\left(\alpha_{i} y_{i}\right)\right|: i \leq m, x^{*} \in \bigcup_{t \geq k} D_{i}\right\}+2 \varepsilon\left\|\left(\alpha_{i}\right)\right\|_{\infty} .
\end{aligned}
$$

We have used that if $j \in I_{0}$, then $j>j_{1}$ and so from the fact that $\left(x_{1}^{*}, \ldots, x_{\ell}^{*}\right)$ is $(\mathcal{D}, M, \sigma)$-admissible and $\max \operatorname{supp} x_{j_{1}}^{*} \geq \min \operatorname{supp} y>k$ we have that $x_{j}^{*} \in D_{t}$ for some $t>k$. Furthermore, the "min $(2, \ell-1)$ " came from

$$
\left|\left\{j \in I_{0}: x_{j}^{*}\left(y_{i_{0}}\right) \neq 0\right\}\right| \leq \min (2, \ell-1) .
$$

Continuing, the right-hand expression in (3) is

$$
\begin{aligned}
& \leq \frac{\left|x_{j_{1}}^{*}(y)\right|}{f(\ell)}+\min (\ell-1,1)\left(1+\frac{1}{f(\ell)}\right)\left\|\left(\alpha_{i}\right)\right\|_{\infty} \\
& \quad+\frac{1}{f(\ell)} \min (m-1, \ell-1) \sup \left\{\left|x^{*}(y)\right|: x^{*} \in \bigcup_{t \geq k} D_{t}\right\} \\
& \quad+\frac{1}{f(\ell)} \min (2, \ell-1)\left\|\left(\alpha_{i}\right)\right\|_{\infty} \sup \left\{\left|x^{*}\left(y_{i}\right)\right|: i \leq m, x^{*} \in \bigcup_{t \geq k} D_{t}\right\} \\
& \quad+2 \varepsilon\left\|\left(\alpha_{i}\right)\right\|_{\infty} .
\end{aligned}
$$

(3) and (4) combined yield $(2.2 .2)$ (using $\frac{\min (2, \ell-1)}{f(\ell)} \leq 1$ ).

b) To deduce (2.2.4) it suffices to prove that given $\varepsilon>0$ and $m \in \mathbb{N}$

$$
\exists \tilde{n}_{1} \in \mathbb{N} \forall n_{1} \geq \tilde{n}_{1} \exists \tilde{n}_{2}>n_{1} \forall n_{2} \geq \tilde{n}_{2} \ldots \exists \tilde{n}_{m}>n_{m-1} \forall n_{m} \geq \tilde{n}_{m}
$$

so that we have

$(*)$ for all $\ell \in \mathbb{N}$ and $x^{*}=\frac{1}{f(\ell)} \sum_{j=1}^{\ell} x_{j}^{*} \in \Gamma_{\ell}$ and $\left(\alpha_{i}\right)_{1}^{m} \subseteq \mathbb{R}$,

$$
\left|x^{*}\left(\sum_{i=1}^{m} \alpha_{i} y_{n_{i}}\right)\right| \leq \frac{1}{f(\ell)}\left\|\sum_{i=1}^{m} \alpha_{i} y_{n_{i}}\right\|_{D}+(\ell+4) \varepsilon\left\|\left(\alpha_{i}\right)\right\|_{\infty}, \quad \text { and }
$$

$(* *)$ for any $\left(\alpha_{i}\right)_{1}^{m} \subseteq \mathbb{R}$

$$
\left\|\sum_{i=1}^{m} \alpha_{i} y_{n_{i}}\right\|_{\Gamma} \leq \max \left\{\left\|\left(\alpha_{i}\right)\right\|_{\infty},\left\|\sum_{i=1}^{m} \alpha_{i} y_{n_{i}}\right\|_{D}\right\}+7 \varepsilon\left\|\left(\alpha_{i}\right)\right\|_{\infty}
$$

To see this, given $m$ and $\varepsilon$, begin by choosing $\tilde{n}_{1}$ so that

$$
\begin{aligned}
& \varepsilon_{\tilde{n}_{1}}<\frac{\varepsilon}{2 m^{2}} \text { and } \\
& \text { if } \ell \in \mathbb{N} \text { with } \frac{\ell}{f(\ell)} \varepsilon_{\tilde{n}_{1}}>\varepsilon, \text { then } \frac{1}{f(\ell)}<\frac{\varepsilon}{m} .
\end{aligned}
$$

Assume $\tilde{n}_{1}<n_{1}<\cdots<\tilde{n}_{i}<n_{i}$ are chosen $\left(n_{j}>\tilde{n}_{j}\right.$ arbitrarily and $\tilde{n}_{j}$ dependent on $\left.n_{j-1}\right)$. Use (2.2.3) to choose $\tilde{n}_{i+1}>n_{i}$ so that: 
(7) for any $n_{i+1} \geq \tilde{n}_{i+1}$ and any $(\mathcal{D}, M, \sigma)$-admissible sequence $\left(x_{1}^{*}, \ldots, x_{j}^{*}\right)$ with $\max \operatorname{supp} x_{j}^{*} \geq \min \operatorname{supp} y_{n_{i+1}}$ and $x^{*} \in D_{t}$ for some $t \geq \sigma\left(x_{1}^{*}, \ldots, x_{j}^{*}\right)$ we have

$$
\left|x^{*}\left(y_{n_{i+1}}\right)\right|<\frac{\varepsilon}{f^{-1}\left(\frac{\max \operatorname{supp} y_{n_{i}}}{\varepsilon}\right)} .
$$

Now let $\ell \in \mathbb{N}, y=\sum_{1}^{m} \alpha_{i} y_{n_{i}}$ and $x^{*}=\frac{1}{f(\ell)} \sum_{j=1}^{\ell} x_{j}^{*} \in \Gamma_{\ell}$. Let $j_{1}$ be defined as in a). From the first inequality in $(2.2 .2)$ we have ( $k$ is replaced by $\tilde{n}_{1}$ )

$$
\begin{aligned}
\left|x^{*}(y)\right| \leq & \frac{1}{f(\ell)}\left|x_{j_{1}}^{*}(y)\right|+\left\|\left(\alpha_{i}\right)\right\|_{\infty} \max _{i \leq m} \sum_{j=j_{1}+1}^{\ell} \frac{\left|x_{j}^{*}\left(y_{n_{i}}\right)\right|}{f(\ell)} \\
& +\frac{m}{f(\ell)} \cdot m \cdot \varepsilon_{\tilde{n}_{1}} \cdot\left\|\left(\alpha_{i}\right)\right\|_{\infty} \\
& +\frac{2}{f(\ell)} \varepsilon_{\tilde{n}_{1}}\left\|\left(\alpha_{i}\right)\right\|_{\infty}+2 \varepsilon\left\|\left(\alpha_{i}\right)\right\|_{\infty} \\
\leq & \frac{1}{f(\ell)}\left|x_{j_{1}}^{*}(y)\right|+\left\|\left(\alpha_{i}\right)\right\|_{\infty} \ell \varepsilon_{\tilde{n}_{1}}+\left\|\left(\alpha_{i}\right)\right\|_{\infty} \cdot 4 \varepsilon
\end{aligned}
$$

where the last inequality uses (5) to deduce $m^{2} \varepsilon_{\tilde{n}_{1}}<\varepsilon$ and the trivial estimate $\frac{2 \varepsilon_{\tilde{n}_{1}}}{f(\ell)}<\varepsilon$. Thus

$$
\left|x^{*}(y)\right| \leq \frac{1}{f(\ell)}\|y\|_{D}+\left\|\left(\alpha_{i}\right)\right\|_{\infty} \varepsilon(\ell+4) .
$$

This completes the proof of $(*)$.

To see $(* *)$ let $\ell \in \mathbb{N}$ and $x^{*}=\frac{1}{f(\ell)} \sum_{j=1}^{\ell} x_{j}^{*} \in \Gamma_{\ell}$ so that for $y=\sum_{1}^{m} \alpha_{i} y_{n_{i}}$,

$$
\|y\|=\|y\|_{\Gamma_{\ell}} \leq\left|x^{*}(y)\right|+\varepsilon\left\|\left(\alpha_{i}\right)\right\|_{\infty} .
$$

Case 1. $\frac{\ell}{f(\ell)} \varepsilon_{\tilde{n}_{1}} \leq \varepsilon$.

From (8) above we have

$$
\begin{aligned}
\left|x^{*}(y)\right| & \leq \frac{1}{f(\ell)}\|y\|_{D}+\left\|\left(\alpha_{i}\right)\right\|_{\infty} \max _{i \leq m} \sum_{j=j_{1}+1}^{\ell} \frac{\left|x_{j}^{*}\left(y_{n_{i}}\right)\right|}{f(\ell)}+\left\|\left(\alpha_{i}\right)\right\|_{\infty} \cdot 4 \varepsilon \\
& \leq \frac{1}{f(\ell)}\|y\|_{D}+\left\|\left(\alpha_{i}\right)\right\|_{\infty}\left[\frac{\ell \varepsilon_{\tilde{n}_{1}}}{f(\ell)}+4 \varepsilon\right] \\
& \leq \frac{1}{f(\ell)}\|y\|_{D}+\left\|\left(\alpha_{i}\right)\right\|_{\infty} \cdot 5 \varepsilon .
\end{aligned}
$$

Case 2. $\frac{\ell}{f(\ell)} \varepsilon_{\tilde{n}_{1}}>\varepsilon$ and hence by $(6), \frac{1}{f(\ell)}<\frac{\varepsilon}{m}$.

Note that

$$
\begin{aligned}
\max _{i \leq m}\left|\alpha_{i}\right|\left|\left(x^{*}-\frac{x_{j_{1}}^{*}}{f(\ell)}\right)\left(y_{i}\right)\right| & \leq\left\|\left(\alpha_{i}\right)\right\|_{\infty}\left(1+\max _{i \leq m} \frac{\left|x_{j_{1}}^{*}\left(y_{i}\right)\right|}{f(\ell)}\right) \\
& \leq\left\|\left(\alpha_{i}\right)\right\|_{\infty}\left(1+\frac{1}{f(\ell)}\right) .
\end{aligned}
$$


Thus using (2.2.2) as in (8) except for this estimate we have

$$
\begin{aligned}
\left|x^{*}(y)\right| & \leq \frac{\|y\|_{D}}{f(\ell)}+\left\|\left(\alpha_{i}\right)\right\|_{\infty}\left(1+\frac{1}{f(\ell)}\right)+\left\|\left(\alpha_{i}\right)\right\|_{\infty} \cdot 4 \varepsilon \\
& \leq\left(\frac{m}{f(\ell)}+1+\frac{1}{f(\ell)}+4 \varepsilon\right)\left\|\left(\alpha_{i}\right)\right\|_{\infty} \\
& <(6 \varepsilon+1)\left\|\left(\alpha_{i}\right)\right\|_{\infty} .
\end{aligned}
$$

We used $\|y\|_{D} \leq m\left\|\left(\alpha_{i}\right)\right\|_{\infty}$. This completes the proof of $(* *)$.

Remark 2.3. Lemma 2.2 also holds in the setting where $\Gamma \equiv \bigcup_{n \geq 2} \Gamma_{n}$ and we shall also use it this way below.

\section{The construction of $X$}

Let $H \subset c_{00} \cap[-1,1]^{\mathbb{N}}$ be a countable set of nonzero elements satisfying the following three properties.:

$\left(\mathrm{H}_{1}\right) H$ is dense in $c_{00}$ with respect to $\|\cdot\|_{\ell_{1}}$,

$\left(\mathrm{H}_{2}\right)$ If $a \in H, I$ is an interval of integers and $I a \neq 0$, then $I a \in H$.

$\left(\mathrm{H}_{3}\right)$ For all $n \in \mathbb{N}, a_{1}<\cdots<a_{n}$ in $H$ we have $\sum_{i=1}^{n} a_{i}, \frac{1}{f(n)} \sum_{i=1}^{n} a_{i}$ and $\frac{1}{n} \sum_{i=1}^{n} a_{i}$ are all in $H$.

Let $M=\left(M_{n}\right) \subseteq \mathbb{N}$ be strictly increasing with $M_{1}=2$. Let

$$
\sigma:\left\{\left(a_{1}, \ldots, a_{n}\right): n \in \mathbb{N}, a_{1}<\cdots<a_{n}, a_{i} \in H \text { for } i \leq n\right\} \rightarrow \mathbb{N}
$$

be an injection satisfying the following four properties:

$\left(\sigma_{1}\right)$ Let $n \in \mathbb{N}$ and $a_{1}<\cdots<a_{n}$ be in $H$. Let $I$ be an interval in $\mathbb{N}$ and suppose $\left[j_{1}, j_{2}\right]=\left\{i: I a_{i} \neq 0, i \leq n\right\} \neq \emptyset$. Then $\sigma\left(I a_{j_{1}}, \ldots, I a_{j_{2}}\right) \leq \sigma\left(a_{1}, \ldots, a_{n}\right)$.

$\left(\sigma_{2}\right)$ For $n \in \mathbb{N}$ and $a_{1}<\cdots<a_{n}$ in $H$,

$$
\max \operatorname{supp} a_{n}<\sigma\left(a_{1}, \ldots, a_{n}\right) \text {. }
$$

$\left(\sigma_{3}\right)$ Let $I_{m}(\sigma)$ be the range of $\sigma$. Then

$$
C_{f}(\sigma) \equiv \sum_{n \in I_{m}(\sigma)} \frac{1}{f(n)}<\infty .
$$

In particular for $1 \leq p<\infty$

$$
C_{p}(\sigma) \equiv \sum_{n \in I_{m}(\sigma)} n^{-1 / p}<\infty
$$

$\left(\sigma_{4}\right)$ For $m \in \operatorname{Im}(\sigma)$ let $\bar{m}$ and $\overline{\bar{m}}$ be the predecessor and successor, respectively, of $m$ in $\operatorname{Im}(\sigma)$. Then, if $\ell \in[1, \bar{m}] \cup[\overline{\bar{m}}, \infty)$,

$$
2\left[\frac{f(m)}{m}+\frac{1}{f(\ell)}+\frac{f(m)}{m} \frac{\min (\ell, m)}{f(\ell)}\right] \leq \begin{cases}\frac{3}{f(\ell)}, & \ell<m, \\ \frac{3 f(m)}{m}, & \ell>m .\end{cases}
$$

We have made no attempt to give a minimal list of necessary conditions but rather we have chosen to list precisely the conditions we will use. It is straightforward to prove that such a set $H$ and function $\sigma$ exist. 
For $\|\cdot\| \in \mathcal{N}$ let $X=\left(c_{00},\|\cdot\|\right)$. For $m \geq 2$ let

$$
A_{m}^{X}=\left\{\frac{1}{m} \sum_{i=1}^{m} a_{i}^{*}: a_{i}^{*} \in H \cap B\left(X^{*}\right) \text { for } i \leq m \text { and } a_{1}^{*}<\cdots<a_{m}^{*}\right\} .
$$

Set $A^{X}=\bigcup_{m \geq 2} A_{m}^{X}, \tilde{A}_{m}^{X}=\bigcup_{n \geq m} A_{n}^{X}$ for $m \geq 2$ and $\tilde{\mathfrak{A}}^{X}=\left(\tilde{A}_{m}^{X}\right)_{m=2}^{\infty}$. Note that $A^{X} \subseteq B\left(X^{*}\right)$. For $m \geq 2$ let

$$
B_{m}^{X}=\left\{\frac{1}{f(m)} \sum_{i=1}^{m} a_{i}^{*}:\left(a_{1}^{*}, \ldots, a_{m}^{*}\right) \subseteq A^{X} \text { is }\left(\tilde{\mathfrak{A}}^{X}, M, \sigma\right) \text {-admissible }\right\} .
$$

Let $B^{X}=\bigcup_{n=2}^{\infty} B_{n}^{X}$ and $\mathfrak{B}^{X}=\left(B_{n}^{X}\right)_{n=2}^{\infty}$. For $m \geq 1$ set

$$
C_{m}^{X}=\left\{\frac{1}{f(m)} \sum_{i=1}^{m} a_{i}^{*}:\left(a_{1}^{*}, \ldots, a_{m}^{*}\right) \subseteq B^{X} \text { is }\left(\mathfrak{B}^{X}, M, \sigma\right) \text {-admissible }\right\} .
$$

Note that $C_{1}^{X}=B^{X}$ and let $C^{X}=\bigcup_{n=1}^{\infty} C_{n}^{X}$.

In the notation of $\S 2$,

$$
B_{n}^{X}=\Gamma_{n}\left(\tilde{\mathfrak{A}}^{X}, M, \sigma\right) \text { and } C_{n}^{X}=\Gamma_{n}\left(\mathfrak{B}^{X}, M, \sigma\right) .
$$

Remark. Any element of $A_{n}^{X}, \tilde{A}_{n}^{X}$ or $B_{n}^{X}$ has at least $n$ nonzero coordinates. Thus this plus $\left(\sigma_{2}\right)$ implies that conditions $(\sigma, \tilde{\mathfrak{A}})$ and $(\sigma, \mathfrak{B})$ hold (see $\left.\S 2\right)$. Also $A_{n}^{X}, B_{n}^{X}, C_{n}^{X} \subseteq[-1,1]^{\mathbb{N}}$ for all $n$.

Proposition 3.1. There exists a norm $\|\cdot\| \in \mathcal{N}$ so that for $X=\left(c_{00},\|\cdot\|\right)$ and all $x \in c_{00}$,

$$
\|x\|=\max \left(\|x\|_{\infty},\|x\|_{C^{X}}\right) .
$$

Moreover the completion of $X$ is reflexive.

Proof. Define $P: \mathcal{N} \rightarrow \mathcal{N}$ by $P\|x\|=\max \left\{\|x\|_{\infty},\|x\|_{C^{X},\|\cdot\|}\right\}$. To see that $P\|\cdot\| \in$ $\mathcal{N}$ we need only note that $\left(e_{i}\right)$ is monotone for $P\|\cdot\|$. This follows from the fact that if $I \subseteq \mathbb{N}$ is an initial interval $\left(I=[1, n]\right.$ for some $n$ ) and $x^{*} \in C_{\ell}^{X}$ (or $A_{\ell}^{X}, B_{\ell}^{X}$ ), then for all $m>n$ there exists $y^{*} \in C_{\ell}^{X}$ with $I y^{*}=I x^{*}$ and $y^{*}(k)=0$ if $n<k \leq m$. This easily established fact uses that $\left(e_{i}\right)$ is a monotone basis for $\|\cdot\|$.

$P$ is order preserving. This follows from the fact that if $|\cdot| \leq\|\cdot\|$ are norms in $\mathcal{N}$, then $C^{X,|\cdot|} \subseteq C^{X,\|\cdot\|}$. Thus by Proposition 2.1 we obtain a norm $\|\cdot\| \in \mathcal{N}$ satisfying (3.1.1).

It remains to show that the completion of $X=\left(c_{00},\|\cdot\|\right)$ is reflexive. To do this we shall prove that $\left(e_{i}\right)$ is shrinking and boundedly complete. If $\left(e_{i}\right)$ were not boundedly complete, then there exists a block basis $\left(x_{i}\right)$ of $\left(e_{i}\right)$ with $\left\|x_{i}\right\| \geq 1$ for all $i$ and $\left\|\sum_{i=1}^{n} x_{i}\right\| \leq K$ for all $n$ and some $K<\infty$. Choose $x_{i}^{*} \in B\left(X^{*}\right) \cap H$ with for all $i, \operatorname{supp} x_{i}^{*} \subseteq\left[\min \operatorname{supp} x_{i}, \max \operatorname{supp} x_{i}\right]$ and $x_{i}^{*}\left(x_{i}\right)>1 / 3$. Indeed using that $\left(e_{i}\right)$ is a monotone basis for $X$ we can produce $y_{i}^{*} \in X^{*}$ with $\left\|y_{i}^{*}\right\| \leq 2$, supp $y_{i}^{*} \subseteq$ $\left[\min \operatorname{supp} x_{i}, \max \operatorname{supp} x_{i}\right]$ and $y_{i}^{*}\left(x_{i}\right)=\left\|x_{i}\right\| \geq 1$. Then we take $x_{i}^{*}$ in $H \cap B\left(X^{*}\right)$ to be an appropriate approximation of $\frac{y_{i}^{*}}{\left\|y_{i}^{*}\right\|}$. Let $\ell>1$ be fixed. Then $a_{1}^{*} \equiv$ $\frac{1}{M_{\ell}} \sum_{1}^{M_{\ell}} x_{i}^{*} \in A_{M_{\ell}}^{X}$. We choose $a_{2}^{*}=\frac{1}{\sigma\left(a_{1}^{*}\right)} \sum_{i=M_{\ell}+1}^{M_{\ell}+\sigma\left(a_{1}^{*}\right)} x_{i}^{*}$ and so forth obtaining ultimately $\left(a_{1}^{*}, \ldots, a_{\ell}^{*}\right)$ which is $\left.\tilde{\mathfrak{A}}, M, \sigma\right)$-admissible. It follows that $\frac{1}{f(\ell)} \sum_{1}^{\ell} a_{\ell}^{*} \in$ 
$B\left(X^{*}\right)$ and for an appropriate $n$

$$
\frac{1}{f(\ell)} \sum_{1}^{\ell} a_{i}^{*}\left(\sum_{j=1}^{n} x_{i}\right) \geq \frac{1}{f(\ell)} \frac{\ell}{3} .
$$

But this cannot be always bounded by $K$.

Finally, we prove that $\left(e_{i}\right)$ is shrinking. If not, there exists a normalized block basis $\left(x_{i}\right)$ of $\left(e_{i}\right)$ and $\delta>0$ so that $\left\|\sum_{i} a_{i} x_{i}\right\|>\delta \sum_{i} a_{i}$ for all $\left(a_{i}\right) \in c_{00}^{+}$(i.e., $a_{i} \geq 0$ for all $i$ ). Using James' argument [J] that $\ell_{1}$ is not distortable for this $\ell_{1}^{+}$-basis we can, by replacing $\left(x_{i}\right)$ by a normalized block basis, assume that for a given $\varepsilon>0,\left\|\sum a_{i} x_{i}\right\|>(1-\varepsilon) \sum a_{i}$ if $\left(a_{i}\right) \in c_{00}^{+}$. If $\tilde{C}$ is the weak ${ }^{*}$ closure of $C^{X} \cup\left\{e_{n}^{*}\right\}_{\mathbb{N}}$, then we may regard $X \subseteq \mathrm{C}(\tilde{C})$, the space of continuous functions on $\tilde{C}$. Let $\mathcal{F}=\left\{F \subseteq \mathbb{N}\right.$ : there exists $x^{*} \in \tilde{C}$ with $x^{*}\left(x_{i}\right)>1-2 \varepsilon$ for all $\left.i \in F\right\}$. $\mathcal{F}$ is a hereditary family of subsets of $\mathbb{N}$ and has the additional property that, if $\left(a_{i}\right) \subseteq c_{00}^{+}$with $\sum_{i} a_{i}=1$, then there exists $F \in \mathcal{F}$ with $\sum_{F} a_{i} \geq \varepsilon$. Indeed let $x^{*} \in \tilde{C}$ with $x^{*}\left(\sum a_{i} x_{i}\right)>1-\varepsilon$ and set $F=\left\{i: x^{*}\left(x_{i}\right)>1-2 \varepsilon\right\}$. Then

$$
1-\varepsilon<x^{*}\left(\sum a_{i} x_{i}\right) \leq(1-2 \varepsilon) \sum_{i \notin F} a_{i}+\sum_{i \in F} a_{i} \leq(1-2 \varepsilon)+\sum_{i \in F} a_{i}
$$

and so $\varepsilon \leq \sum_{i \in F} a_{i}$.

Ptak's theorem ([$]$, see also $[\mathrm{BHO}])$ yields that there exists a subsequence $N$ of $\mathbb{N}$ so that $F \in \mathcal{F}$ for all $F \subseteq N$. Thus relabeling $\left(x_{i}\right)_{N}$ as $\left(x_{i}\right)$ we have that for all $n$ there exists $x_{n}^{*} \in C^{X} \cup\left\{e_{m}^{*}\right\}_{\mathbb{N}}$ so that $x_{n}^{*}\left(x_{i}\right)>1-2 \varepsilon$ for $i \leq n$. Now for $n \geq 2$ of course we have $x_{n}^{*} \in C^{X}$. Suppose that $x_{n}^{*}=\frac{1}{f\left(\ell_{n}\right)} \sum_{1}^{\ell_{n}} b_{j}^{*} \in C_{\ell(n)}^{X}$. If $\ell(n) \geq 2$, then since $f\left(\ell_{n}\right)>1$ it must be true that $b_{j}^{*}\left(x_{i}\right) \neq 0$ for at least two $j$ 's for each $i \leq n$ (provided $\varepsilon>0$ satisfies $\frac{1}{f(2)}<1-2 \varepsilon$ ). Thus if $\ell(n) \geq 2$ for all $n$ we have that $\ell(n) \rightarrow \infty$. But then $x_{n}^{*}\left(x_{1}\right) \rightarrow 0$ as $n \rightarrow \infty$, a contradiction. We are left with the case (passing to a subsequence) where $\ell(n)=1$ for all $n$ and so $x_{n}^{*} \in C_{1}^{X}=B^{X}$. But then $x_{n}^{*} \in B_{j(n)}^{X}$ where $j(n) \geq 2$ for all $n$ and so by the argument we just gave adapted with minor notational changes, we obtain $x_{n}^{*}\left(x_{1}\right) \rightarrow 0$, a contradiction.

Henceforth, $X=\left(c_{00},\|\cdot\|\right)$ shall denote the normed space obtained in Proposition 3.1. We shall write $\tilde{A}_{m}, A_{m}, B_{m}, C_{m}, B, C, \tilde{\mathfrak{A}}, \mathfrak{A}$ and $\mathfrak{B}$ rather than $\tilde{A}_{m}^{X}, A_{m}^{X}, \ldots$ etc.

We can now state the main result of this paper.

Theorem 3.2. Let $\left(y_{i}\right)$ be any block basis of $\left(e_{i}\right)$ in $X$. Let $\left(f_{i}\right)_{1}^{n}$ be any finite monotone basis. Then $\left(f_{i}\right)_{1}^{n}$ is block finitely represented in $\left(y_{i}\right)$.

First we reduce the proof to the consideration of a special class of monotone bases. For $n \in \mathbb{N}$ let $\left(S^{n}(i, j)\right)_{i, j=1}^{n}$ be linearly independent vectors in some linear space. For $(a(i, j))_{i, j=1}^{n} \subseteq \mathbb{R}$ define

$$
\left\|\sum_{i, j=1}^{n} a(i, j) S^{n}(i, j)\right\|=\max _{j \leq n} \max _{k \leq n}\left|\sum_{i=1}^{k} a(i, j)\right| .
$$

$\left(S^{n}(i, j)\right)_{i, j=1}^{n}$ is a normalized monotone basis under the norm when ordered lexicographically: $S^{n}(1,1), S^{n}(1,2), \ldots, S^{n}(1, n), S^{n}(2,1), \ldots, S^{n}(n, n)$. For fixed $j \leq n$, $\left(S^{n}(i, j)\right)_{i=1}^{n}$ is 1-equivalent to the usual summing basis of length $n$. 
Proposition 3.3. If for all $n \in \mathbb{N},\left(S^{n}(i, j)\right)_{i, j=1}^{n}$ is block finitely represented in a basic sequence $\left(y_{i}\right)$, then every finite monotone basis is block finitely represented in $\left(y_{i}\right)$.

Proof. Let $\left(z_{i}\right)_{i=1}^{m}$ be a finite monotone basis for a space $Z$. We may assume that $Z$ is a subspace of $\ell_{\infty}^{n}$ for some $n$. For $i \leq m$ write $z_{i}=(z(i, j))_{j=1}^{n} \in \ell_{\infty}^{n}$, i.e., $z_{i}(j)=z(i, j)$. For $i \leq m$ set $w_{i}=\sum_{j=1}^{n} z(i, j) S^{n}(i, j)$. We claim that for all $\left(b_{i}\right)_{1}^{m} \subseteq \mathbb{R}$

$$
\left\|\sum_{i=1}^{m} b_{i} w_{i}\right\|=\left\|\sum_{i=1}^{m} b_{i} z_{i}\right\|_{\ell_{\infty}^{n}} .
$$

Indeed since $\left(z_{i}\right)_{1}^{m}$ is monotone,

$$
\begin{aligned}
\left\|\sum_{i=1}^{m} b_{i} z_{i}\right\|_{\ell_{\infty}^{n}} & =\max _{k \leq m}\left\|\sum_{i=1}^{k} b_{i} z_{i}\right\|_{\ell_{\infty}}^{n}=\max _{k \leq m} \max _{j \leq n}\left|\sum_{i=1}^{k} b_{i} z(i, j)\right| \\
& =\left\|\sum_{i=1}^{m} \sum_{j=1}^{n} b_{i} z(i, j) S(i, j)\right\|=\left\|\sum_{i=1}^{m} b_{i} w_{i}\right\| . \square
\end{aligned}
$$

Thus we are reduced to proving that for all $n,\left(S^{n}(i, j)\right)_{i, j=1}^{n}$ is block finitely represented in every block basis of $\left(e_{i}\right)$. Next we state our Main Lemma and show how it yields the theorem. The Main Lemma will be proved in $\S 4$.

Main Lemma 3.4. Let $Y$ be a block subspace of $X$. Then there exists a constant $C=C(Y) \geq 1$ so that for all $\varepsilon>0$ there exists $m_{0} \in \mathbb{N}$ with the following property $(*)$.

(*) For any $k \in \mathbb{N}, \delta>0$ and $m \in \operatorname{Im}(\sigma)$ with $m \geq m_{0}$ and $\frac{f(m)}{m}<\frac{\delta}{8}$ there exists $y \in Y$ with the following properties:

a) $e_{k}<y$.

b) $\|y\| \leq C$.

c) there exists $y^{*} \in B_{m}$ with $e_{k}^{*}<y^{*}$ and $y^{*}(y)>1$.

d) i) $\|y\|_{A_{\ell}}<\varepsilon$ for all $\ell \in \mathbb{N}$ with $\ell \geq m_{0}$,

ii) $\|y\|_{B_{\ell}}<\varepsilon$ for all $\ell \in \mathbb{N}$ with $\ell \in\left[m_{0}, \bar{m}\right] \cup[\overline{\bar{m}}, \infty)$,

where $\bar{m}$ and $\overline{\bar{m}}$ are the predecessor of $m$ and successor of $m$ in $I_{m}(\sigma)$.

e) i) If $\left(x_{1}^{*}<\cdots<x_{j}^{*}\right)$ is $(\tilde{\mathfrak{A}}, M, \sigma)$-admissible with maxupp $x_{j}^{*} \geq$ min supp $y$, then

$$
\left|x^{*}(y)\right|<\delta \text { for all } x^{*} \in \bigcup_{m \geq \sigma\left(x_{1}^{*}, \ldots, x_{j}^{*}\right)} A_{m} .
$$

ii) If $\left(y_{1}^{*}, \ldots, y_{j}^{*}\right)$ is $(\mathfrak{B}, M, \sigma)$-admissible with $\max \operatorname{supp} y_{j}^{*} \geq \min \operatorname{supp} y$, then

$$
\left|x^{*}(y)\right|<\delta \text { for all } y^{*} \in \bigcup_{m \geq \sigma\left(y_{1}^{*}, \ldots, y_{j}^{*}\right)} B_{m} .
$$


Before proving Theorem 3.2 we present an elementary but often used lemma.

Lemma 3.5. If $x_{1}<\cdots<x_{m}$ is a sequence in $B(X), \ell \in \mathbb{N}$, $\ell \geq 2$, then

$$
\begin{aligned}
&\left\|\sum_{i=1}^{m} x_{i}\right\|_{A_{\ell}} \leq \frac{1}{\ell} \max \left\{\sum_{j=1}^{\ell}\left\|\sum_{i=k_{j-1}}^{k_{j}} x_{i}\right\|: 1 \leq k_{0} \leq k_{1} \leq \cdots \leq k_{\ell} \leq m\right\} \\
& \leq 1+\frac{1}{\ell} \max \left\{\sum_{j=1}^{\ell^{\prime}}\left\|\sum_{i=k_{j-1}+1}^{k_{j}} x_{i}\right\|: 0 \leq k_{0}<k_{1}<\cdots<k_{\ell^{\prime}} \leq m\right. \\
&\left.\quad \text { where } \ell^{\prime} \leq \min (\ell, m)\right\}
\end{aligned}
$$

Proof. Let $x^{*}=\frac{1}{\ell} \sum_{j=1}^{\ell} x_{j}^{*}$ where $x_{1}^{*}<\cdots<x_{\ell}^{*}$ are in $B\left(X^{*}\right) \cap H$ and set $x=$ $\sum_{1}^{m} x_{i}$. Let $k_{0}=1$ and for $j \geq 1$ set

$$
k_{j}=\min \left\{i \geq 1: \max \operatorname{supp} x_{j}^{*} \leq \max \operatorname{supp} x_{i}\right\}
$$

if such an $i$ exists and $k_{j}=m$ otherwise. Then $1=k_{0} \leq k_{1} \leq \cdots \leq k_{\ell} \leq m$ and

$$
\begin{aligned}
\left|x^{*}(x)\right| & =\frac{1}{\ell}\left|\sum_{j=1}^{\ell} x_{j}^{*}(x)\right|=\frac{1}{\ell}\left|\sum_{j=1}^{\ell}\left(\sum_{i=k_{j-1}}^{k_{j}} x_{j}^{*}\left(x_{i}\right)\right)\right| \\
& \leq \frac{1}{\ell} \sum_{j=1}^{\ell}\left\|\sum_{i=k_{j-1}}^{k_{j}} x_{i}\right\| \\
& \leq \frac{1}{\ell}\left[\sum_{j=1}^{\ell}\left\|x_{k_{j-1}}\right\|+\sum_{j=1}^{\ell}\left\|\sum_{i=k_{j-1}+1}^{k_{j}} x_{i}\right\|\right] \\
& \leq 1+\frac{1}{\ell} \max \left\{\sum_{j=1}^{\ell^{\prime}}\left\|\sum_{i=k_{j-1}^{\prime}+1}^{k_{j}^{\prime}} x_{i}\right\|: \ell^{\prime} \leq \min (\ell, m),\right. \\
& \left.0 \leq k_{0}^{\prime}<k_{1}^{\prime}<\cdots<k_{\ell^{\prime}}^{\prime} \leq m\right\}
\end{aligned} .
$$

Corollary 3.6. Let $1 \leq p<\infty, k \in \mathbb{N}$ and let $x \in X$ be an $\ell_{p}^{k}$-average with constant 2. Then for $\ell \geq 2$,

$$
\|x\|_{A_{\ell}} \leq k^{-1 / p}+2 \ell^{-1 / p} .
$$

Proof. Let $x=k^{-1 / p} \sum_{i=1}^{k} x_{i}$ where $x_{1}<\cdots<x_{k}$ is a normalized sequence 2equivalent to the unit vector basis of $\ell_{p}^{k}$. By Lemma 3.5 there exist $0 \leq k_{0}<k_{1}<$ 
$\cdots<k_{\ell^{\prime}} \leq k$ for some $\ell^{\prime} \leq \min (\ell, k)$ satisfying

$$
\begin{aligned}
\|x\|_{A_{\ell}} & \leq k^{-1 / p}\left[1+\frac{1}{\ell} \sum_{j=1}^{\ell^{\prime}}\left\|\sum_{i=k_{j-1}+1}^{k_{j}} x_{i}\right\|\right] \\
& \leq k^{-1 / p}+\ell^{-1} k^{-1 / p} \sum_{j=1}^{\ell^{\prime}} 2\left(k_{j}-k_{j-1}\right)^{1 / p} \\
& \leq k^{-1 / p}+2 \ell^{-1} k^{-1 / p} \ell^{\prime}\left[k / \ell^{\prime}\right]^{1 / p} \\
& \leq k^{-1 / p}+2 \ell^{-1} k^{-1 / p} \ell^{1-1 / p} k^{1 / p} \\
& =k^{-1 / p}+2 \ell^{-1 / p} .
\end{aligned}
$$

We have used the concavity of the function $x^{1 / p}$ to obtain the third inequality.

Proof of Theorem 3.2. Fix $n$ and a block basis $\left(y_{i}\right)$ of $\left(e_{i}\right)$ in $X$. We shall prove that $\left(S^{n}(i, j)\right)_{i, j=1}^{n}$ is block finitely represented in $\left(y_{i}\right)$. Let $\varepsilon>0$ satisfy

$$
\gamma \equiv \frac{1}{f(2)}\left[1+\frac{\varepsilon}{1-\varepsilon}\right]<1 \text { with } 2 \varepsilon<\gamma .
$$

Let $C$ be as in Lemma 3.4 for $Y=\left[\left(y_{i}\right)\right]$. We next choose $k, \ell_{0} \in \mathbb{N}$ and $\varepsilon_{0}>0$ to satisfy

$$
\begin{aligned}
& \frac{f(k)}{f(n k)}>1-\varepsilon \text { and } 6 C n \frac{f(k)}{k}<\varepsilon, \\
& \frac{4 C n^{3} k^{2}}{f\left(\ell_{0}\right)}<\varepsilon, \text { and } \\
& 6 \ell_{0} k n^{2} \varepsilon_{0}<\varepsilon .
\end{aligned}
$$

Then we let $m_{0} \geq M_{n k}$ be given by Lemma 3.4 for $\varepsilon_{0} / 2$. Let $P=\{(i, j, s)$ : $1 \leq i, j \leq n, 1 \leq s \leq k\}$ be ordered lexicographically. Using $(*)$ in Lemma 3.4 we will recursively choose for each $(i, j, s) \in P$ a block $y(i, j, s) \in \operatorname{span}\left(y_{t}\right), y^{*}(i, j, s) \in$ $X^{*} \cap c_{00}$ and an interval $I(i, j, s)$ in $\mathbb{N}$ and $m(i, j, s) \in \mathbb{N}$ to satisfy conditions (4)-(11):

(4) $\operatorname{supp} y(i, j, s) \subseteq I(i, j, s), \operatorname{supp} y^{*}(i, j, s) \subseteq I(i, j, s)$, and $I(i, j, s)<I\left(i^{\prime}, j^{\prime}, s^{\prime}\right)$ if $(i, j, s)<\left(i^{\prime}, j^{\prime}, s^{\prime}\right)$.

(5) $\|y(i, j, s)\| \leq C$,

(6) $y^{*}(i, j, s)(y(i, j, s))=1$,

(7) $y^{*}(i, j, s) \in B_{m(i, j, s)}$ and for each $j \leq n$ the family $\left(y^{*}(i, j, s)\right)_{i \leq n, s \leq k}$ is $(\mathfrak{B}, M, \sigma)$-admissible (ordered lexicographically),

(8) a) $m(i, j, s) \geq m_{0}$ and if $(i, s) \neq(1,1)$, then $m(i, j, s)=\sigma\left(y^{*}(r, j, t):(r, j, t)<\right.$ $(i, j, s))$ and

$$
\frac{f(m(i, j, s))}{m(i, j, s)} \leq \frac{1}{8} \frac{\varepsilon_{0}}{f^{-1}\left(\frac{1}{\varepsilon_{0}} \max \operatorname{supp} y\left(i^{\prime}, j, s^{\prime}\right)\right)}
$$

where $\left(i^{\prime}, s^{\prime}\right)$ is the predecessor of $(i, s)$, b) $m(i, j, s) \neq m(r, \ell, t)$ if $(i, j, s) \neq(r, \ell, t)$.

(9) $\|y(i, j, s)\|_{A_{\ell}}<\varepsilon_{0}$ if $\ell \geq m_{0}$,

(10) $\|y(i, j, s)\|_{B_{\ell}}<\varepsilon_{0}$ if $\ell \in\left[m_{0}, \bar{m}\right] \cup[\overline{\bar{m}}, \infty)$ where $\bar{m}$ and $\overline{\bar{m}}$ are the predecessor and successor of $m(i, j, s)$ in $\operatorname{Im}(\sigma)$. 
(11) If $(i, s) \neq(1,1)$ and $\left(i^{\prime}, s^{\prime}\right)$ is the predecessor of $(i, s)$, then for any $(\mathfrak{B}, M, \sigma)$ or $(\tilde{\mathfrak{A}}, M, \sigma)$-admissible sequence $\left(x_{1}^{*}, \ldots, x_{j_{0}}^{*}\right)$ with

$$
\max \operatorname{supp} x_{j_{0}}^{*} \geq \min \operatorname{supp} y(i, j, s) \text {, }
$$

then

$$
\left|x^{*} y(i, j, s)\right|<\frac{\varepsilon_{0}}{f^{-1}\left(\frac{1}{\varepsilon_{0}} \max \operatorname{supp} y\left(i^{\prime}, j, s^{\prime}\right)\right)} \text { for } x^{*} \in \bigcup_{t \geq \sigma\left(x_{1}^{*}, \ldots, x_{j_{0}}^{*}\right)}\left(B_{t} \cup A_{t}\right) .
$$

Let $(i, j, s) \in P$ and assume that $y(r, \ell, t), y^{*}(r, \ell, t), I(r, \ell, t)$ and $m(r, \ell, t)$ have been selected for all $(r, \ell, t)<(i, j, s)$ so that $(4)-(11)$ are satisfied for all such $(r, \ell, t)$. Furthermore, assume that by letting

$$
m(i, j, s) \equiv \sigma\left(y^{*}(r, j, t):(r, t)<(i, s)\right)
$$

(take $\left.m(1,1,1)=m_{0}\right)(8)$ is satisfied as well for $(i, j, s)$. We will have to choose $y(i, j, s), y^{*}(i, j, s), I(i, j, s)$ and $m\left(i^{\prime \prime}, j^{\prime \prime}, s^{\prime \prime}\right),\left(i^{\prime \prime}, j^{\prime \prime}, s^{\prime \prime}\right)$ being the successor of $(i, j, s)$, so that (4)-(11) are satisfied and so that (8) is satisfied for $\left(i^{\prime \prime}, j^{\prime \prime}, s^{\prime \prime}\right)$.

We then apply $(*)$ of Lemma 3.4 for the following parameters. If $(i, j, s)=$ $(1,1,1)$ we let $k=k(1,1,1)=m_{0}$. Otherwise set

$$
k=k(i, j, s)=\max \operatorname{supp} y^{*}\left(i_{0}, j_{0}, s_{0}\right) \vee \max \operatorname{supp} y\left(i_{0}, j_{0}, s_{0}\right)
$$

where $\left(i_{0}, j_{0}, s_{0}\right)$ is the predecessor of $(i, j, s)$ in $P$. If $(i, s)=(1,1)$, we take $\delta=\varepsilon_{0}$ and $m_{0} \leq m \equiv m(i, j, s) \in \operatorname{Im}(\sigma)$ to satisfy $\frac{f(m)}{m}<\frac{\delta}{8}$ and to be distinct from all $m(r, \ell, t)$ 's previously chosen. If $(i, s) \neq(1,1)$, we take

$$
\delta=\frac{\varepsilon_{0}}{f^{-1}\left(\frac{1}{\varepsilon_{0}} \max \operatorname{supp} y\left(i^{\prime}, j, s^{\prime}\right)\right)}
$$

where $\left(i^{\prime}, s^{\prime}\right)$ is the predecessor of $(i, s)$ and $m \equiv m(i, j, s) \equiv \sigma\left(y^{*}(r, j, t):(r, t)<\right.$ $(i, s))$. Note that in this case we have by our hypothesis (8) that $\frac{f(m)}{m}<\frac{\delta}{8}$ and so $(*)$ of Lemma 3.4 does apply to these parameters:

$$
\left(\varepsilon, m_{0}, k, \delta, m\right)=\left(\varepsilon_{0}, m_{0}, k(i, j, s), \delta, m(i, j, s)\right) .
$$

We thus obtain $\tilde{y}(i, j, s)>e_{k(i, j, s)}$ which satisfies $\|\tilde{y}(i, j, s)\| \leq C$ and both (9), (10) and (11) hold for $\tilde{y}(i, j, s)$ replacing $y(i, j, s)$.

Furthermore, there exists $y^{*} \in B_{m(i, j, s)}$ (and thus infinitely many such $y^{*}$ 's) satisfying $y^{*}(\tilde{y}(i, j, s))>1$ and $y^{*}>e_{k(i, j, s)}^{*}$. In particular, we can choose $y^{*}(i, j, s) \in$ $B_{m(i, j, s)}$ to be one of these $y^{*}$ 's so that, in addition, if $(i, s) \neq(n, k)$ and $\left(i^{\prime \prime}, s^{\prime \prime}\right)$ is the successor of $(i, s)$, then $m\left(i^{\prime \prime}, j, s^{\prime \prime}\right) \equiv \sigma\left(y^{*}(r, j, t):(r, t) \leq(i, s)\right)$ also satisfies the condition in (8).

We then set $y(i, j, s)=\frac{\tilde{y}(i, j, s)}{y^{*}(\tilde{y}(i, j, s))}$ and $I(i, j, s)=(k(i, j, s), \max \operatorname{supp} y(i, j, s) \vee$ $\left.\operatorname{maxsupp} y^{*}(i, j, s)\right)$. This completes the construction of the $y(i, j, s), y^{*}(i, j, s)$, $I(i, j, s)$ and $m(i, j, s)$ satisfying (4)-(11).

For $1 \leq i, j \leq n$ define $y(i, j)=\frac{f(k)}{k} \sum_{s=1}^{k} y(i, j, s)$. From (4) it follows that $\{y(i, j): 1 \leq i, j \leq n\}$ is a block basis of $\left(y_{t}\right)$. Let $(a(i, j))_{i, j \leq n} \subseteq \mathbb{R}$ with $\left\|\sum_{i, j} a(i, j) S^{n}(i, j)\right\|=1$. We shall first show that for $y=\sum_{i, j} a(i, j) y(i, j)$, $\|y\| \geq 1-\varepsilon$. Fix $j_{0}, \ell \leq n$ with $1=\sum_{i=1}^{\ell} a\left(i, j_{0}\right)$ (if the sum is -1 we replace all 
$a(i, j)$ 's by $-(a(i, j))$. Define

$$
y^{*}=\frac{1}{f(\ell k)} \sum_{i=1}^{\ell} \sum_{s=1}^{k} y^{*}\left(i, j_{0}, s\right) .
$$

By (7) we have $y^{*} \in C_{\ell k}$ and furthermore

$$
y^{*}(y)=\frac{1}{f(\ell k)} \frac{f(k)}{k} \sum_{i=1}^{\ell} \sum_{s=1}^{k} a\left(i, j_{0}\right)
$$

by (4) and (6). But this

$$
=\frac{1}{f(\ell k)} \frac{f(k)}{k} \cdot k=\frac{f(k)}{f(\ell k)}>1-\varepsilon
$$

by (1). It remains to prove that $\|y\| \leq 1+\varepsilon$.

Claim 1. Let $x^{*}=\frac{1}{f(r)} \sum_{t=1}^{r} x_{t}^{*} \in C_{r}$ with $r \geq 2$. Then

$$
\left|x^{*}(y)\right| \leq 1+\varepsilon \text { or }\left|x^{*}(y)\right| \leq \gamma\|y\| .
$$

Case 1. $r \geq \ell_{0}$.

For $j \leq n$, the family $\left\{C^{-1} y(i, j, s): i \leq n, s \leq k\right\}$ satisfies the conditions of Lemma 2.2a) for $(k, m, \varepsilon)=\left(m_{0}, n k, \varepsilon_{0}\right)$ and $(\mathcal{D}, M, \sigma)=(\mathfrak{B}, M, \sigma)$ or $(\tilde{\mathfrak{A}}, M, \sigma)$. Since $\|(a(i, j))\|_{\infty} \leq 2$ we deduce from the second inequality in (2.2.2) that for $j \leq n$.

$$
\begin{aligned}
\left|\sum_{i=1}^{n} \sum_{s=1}^{k} a(i, j) x^{*} y(i, j, s)\right| \leq & \frac{1}{f(r)} 2 C n k+\frac{\min (r, k n)}{f(r)} 2 C n k \\
& +2 C\left[1+\frac{1}{f(r)}+2 \varepsilon_{0}+1\right] \\
\leq & \frac{1}{f(r)} 4 C n^{2} k^{2}+6 C .
\end{aligned}
$$

It follows that since $r \geq \ell_{0}$

$$
\begin{aligned}
\left|x^{*}(y)\right| & \leq \frac{f(k)}{k} \sum_{j=1}^{n}\left|\sum_{i=1}^{n} \sum_{s=1}^{k} a(i, j) x^{*} y(i, j, s)\right| \\
& \leq \frac{4 C n^{3} k^{2}}{f(r)}+\frac{6 C n f(k)}{k}<2 \varepsilon<\gamma\|y\| .
\end{aligned}
$$

by (1) and (2) and our choice of $\varepsilon$ and $\gamma$.

Case 2. $r \leq \ell_{0}$.

For $t \leq r$ let $x_{t}^{*} \in B_{m_{t}}$. It may be that $m_{t}=m(i, j, s)$ for some $t>1$ and $(i, j, s) \in P$ with $(i, s) \neq(1,1)$. In this case let $t_{1}$ be the maximum of such $t$ 's and note that

$$
m_{t_{1}}=\sigma\left(x_{1}^{*}, \ldots, x_{t_{1}-1}^{*}\right)=m\left(i_{1}, j_{1}, s_{1}\right)
$$

for some $\left(i_{1}, j_{1}, s_{1}\right) \in P$ with $\left(i_{1}, s_{1}\right) \neq(1,1)$. Also then

$$
m_{t_{1}}=\sigma\left(y^{*}\left(i, j_{1}, s\right):(i, s)<\left(i_{1}, s_{1}\right)\right) \text {. }
$$


By the injectivity of $\sigma$ we deduce that

$$
\left(x_{1}^{*}, \ldots, x_{t_{1}-1}^{*}\right)=\left(y^{*}\left(1, j_{1}, 1\right), y^{*}\left(1, j_{1}, 2\right), \ldots, y^{*}\left(i_{0}, j_{1}, s_{0}\right)\right)
$$

where $\left(i_{0}, s_{0}\right)$ is the predecessor of $\left(i_{1}, s_{1}\right)$. Furthermore $m_{t} \neq m(i, j, s)$ for all $(i, j, s) \in P$ and $m_{t}>m_{t_{1}} \geq m_{0}$ if $t>t_{1}$. Thus by (10) we have that for all $(i, j, s) \in P,\left|x_{t}^{*}(y(i, j, s))\right| \leq \varepsilon_{0}$ if $t>t_{1}$ or if $t=t_{1}$ and $(i, j, s) \neq\left(i_{1}, j_{1}, s_{1}\right)$.

From these observations we obtain

$$
\begin{aligned}
\left|x^{*}(y)\right| \leq & \frac{1}{f(r)}\left|\sum_{i=1}^{i_{0}-1} a\left(i, j_{1}\right) f(k)+a\left(i_{0}, j_{1}\right) \sum_{s=1}^{s_{0}} \frac{f(k)}{k}\right| \\
+ & \frac{1}{f(r)}\left|x_{t_{1}}^{*}(y)\right|+\frac{1}{f(r)} \sum_{t=t_{1}+1}^{r}\left|x_{t}^{*}(y)\right| \\
\leq & \max \left\{\left|\sum_{i=1}^{i_{0}-1} a\left(i, j_{1}\right)\right|,\left|\sum_{i=1}^{i_{0}} a\left(i, j_{1}\right)\right|\right. \\
+ & \left.\frac{1}{f(r)}\left|x_{t_{1}}^{*}(y)\right|+\frac{1}{f(r)} \frac{f(k)}{k} \cdot 2 \cdot n^{2} k \varepsilon_{0} r\right\} .
\end{aligned}
$$

The first term in the last inequality is obtained by noticing that if $i_{0}>1$, then necessarily $r \geq k$ while if $i_{0}=1$, then

$$
\frac{1}{f(r)} \sum_{s=1}^{s_{0}} \frac{f(k)}{k}=\frac{f(k) s_{0}}{f(r) k} \leq \frac{f(k) s_{0}}{f\left(s_{0}\right) k}
$$

since $r \geq s_{0}$. The latter is not bigger than 1 since $s_{0} \leq k$. Thus

$$
\begin{aligned}
\left|x^{*}(y)\right| \leq 1 & +\frac{1}{f(r)} \frac{2 f(k)}{k}\left|x_{t_{1}}^{*} y\left(i_{1}, j_{1}, s_{1}\right)\right| \\
& +\frac{1}{f(r)} \frac{2 f(k)}{k} n^{2} k \varepsilon_{0}+\frac{2 n^{2} \varepsilon_{0} r f(k)}{f(r)} \\
< & +\frac{1}{f(r)}\left[\frac{2 C f(k)}{k}+2 n^{2} f(k) \varepsilon_{0}+2 n^{2} f(k) \varepsilon_{0} r\right] \\
<1 & +\varepsilon \text { using } r \leq \ell_{0},(1) \text { and }(3) .
\end{aligned}
$$

It remains to check the case in which for every $j \in\{1, \ldots, n\}$ and every $t>1$, $m_{t} \neq m(i, j, s)$ whenever $(i, s) \neq(1,1)$. In that case we obtain from (10) for $j \in\{1, \ldots, n\}$ that $\left|x_{t}^{*} y(i, j, s)\right|<\varepsilon_{0}$ whenever $(i, s) \neq(1,1)$ and $t>t_{0}$ where $t_{0}$ is the smallest $t$ for which $\max \operatorname{supp} x_{t}^{*} \geq m_{0}\left(\right.$ note that $m_{t_{0}+1}=\sigma\left(x_{1}^{*}, \ldots, x_{t_{0}}^{*}\right)>$ $\left.\max \operatorname{supp} x_{t_{0}}^{*} \geq m_{0}\right)$. 
Thus we get

$$
\begin{aligned}
\left|x^{*}(y)\right| & =\frac{1}{f(r)}\left|x_{t_{0}}^{*}(y)+\sum_{t=t_{0}+1}^{r} x_{t}^{*}\left(\sum_{i, j=1}^{n} a(i, j) \frac{f(k)}{k} \sum_{s=1}^{k} y(i, j, s)\right)\right| \\
& \leq \frac{\|y\|}{f(r)}+2 n^{2} r f(k) \frac{\varepsilon_{0}}{f(r)} \\
& \leq \frac{\|y\|}{f(r)}+2 n^{2} \ell_{0} k \frac{\varepsilon_{0}}{f(r)} \\
& \leq \frac{1}{f(r)}(\|y\|+\varepsilon), \quad \text { by }(3) \\
& \leq \gamma\|y\|,
\end{aligned}
$$

by the choice of $\gamma$ and the fact that $\|y\|>1-\varepsilon$. This completes Claim 1 .

Claim 2. Let $x^{*}=\frac{1}{f(r)} \sum_{i=1}^{r} x_{i}^{*} \in B_{r}$ with $r \geq 2$. Then $\left|x^{*}(y)\right| \leq \gamma\|y\|$.

Indeed the case $r>\ell_{0}$ is handled exactly the same way as Case 1 in Claim 1. If $r \leq \ell_{0}$ let $t_{0} \in \mathbb{N}$ be minimal so that $\max \operatorname{supp} x_{t_{0}}^{*} \geq m_{0}$. Let $x_{t}^{*} \in A_{s_{t}}$. For $t>t_{0}$, $s_{t}>m_{0}$ by $\left(\sigma_{2}\right)$ and thus by $(9),\left|x_{t}^{*} y(i, j, s)\right|<\varepsilon_{0}$ for $(i, j, s) \in P$. Since $e_{m_{0}}<y$ we deduce, using (3), that

$$
\begin{aligned}
\left|x^{*}(y)\right| & \leq \frac{\left|x_{t_{0}}^{*}(y)\right|}{f(r)}+\frac{1}{f(r)} \ell_{0} n^{2} k \frac{f(k)}{k} \varepsilon_{0} \\
& <\frac{\|y\|+\varepsilon}{f(r)} \leq \gamma\|y\| .
\end{aligned}
$$

By the definition of $\|\cdot\|$ in $X$ we obtain $\|y\| \leq 1+\varepsilon$.

The proof of Theorem 3.2 yields the following corollary. Recall [MMT] that, if $Y$ has a basis $\left(y_{i}\right), n \in \mathbb{N}$ and $\left(x_{i}\right)_{1}^{n}$ is a normalized monotone basis, then $\left(x_{i}\right)_{1}^{n} \in\{Y\}_{n}$ if $\forall \varepsilon>0$

$$
\begin{gathered}
\forall k_{1} \exists \ell_{1}>k_{1} \exists z_{1} \in \operatorname{span}\left(y_{i}\right)_{k_{1}}^{\ell_{1}} \forall k_{2}>\ell_{1} \exists \ell_{2}>k_{2} \exists z_{2} \in \operatorname{span}\left(y_{i}\right)_{k_{2}}^{\ell_{2}} \ldots \forall k_{n} \\
>\ell_{n-1} \exists \ell_{n}>k_{n} \exists z_{n} \in \operatorname{span}\left(y_{i}\right)_{k_{n}}^{\ell_{n}} \text { with }\left(z_{i}\right)_{1}^{n} 1+\varepsilon \text {-equivalent to }\left(x_{i}\right)_{1}^{n} .
\end{gathered}
$$

Corollary 3.7. For all block subspaces $Y$ of $X$ and for all $n,\{Y\}_{n}$ is the set of all normalized monotone bases of length $n$.

\section{Proof of the Main Lemma}

Since the proof of Lemma 3.4 is quite technical, we first outline the argument. Let $Y$ be an arbitrary block subspace of $X$.

Step 1 . We first show that for some $1 \leq p<\infty, \ell_{p}$ is block finitely represented in $Y$. Indeed Krivine's theorem insures that there is a $p \in[1, \infty]$ so that $\ell_{p}$ is block finitely representable in $Y$. Secondly, we will observe (Lemma 4.1) that if $p=\infty$, then blocks of certain $\ell_{\infty}$-averages will produce for a given $k \in \mathbb{N}$ and $\varepsilon>0$ a sequence of length $k$ which is $(1+\varepsilon)$-equivalent to the $\ell_{1}^{k}$-unit vector basis.

Step 2 . Let $1 \leq p<\infty$ be as found in Step 1 . We first estimate the $\|\cdot\|_{B_{\ell}}$ and $\|\cdot\|_{A_{\ell}}$-norm of linear combination of certain $\ell_{p}$-averages (Lemma 4.3). Then we consider a sequence $\left(y_{i}\right)$, where $y_{i}$ is an $\ell_{p}^{k_{i}}$-average of constant $\left(1+\varepsilon_{i}\right)$ with $k_{i} \uparrow \infty$ and $\varepsilon_{i} \downarrow 0$. Let $E$ be a spreading model of a subsequence of $\left(y_{i}\right)$. 
Either $c_{0}$ is block finitely representable in $E$. In that case we will (Lemma 4.5) not only deduce that $c_{0}$ is block finitely representable in $\left[y_{i}\right]$ but also that we can choose for any $\varepsilon>0$ and $k \in \mathbb{N}$ an $\ell_{\infty}^{k}$-average $x$ of constant $(1+\varepsilon)$, so that for any $(\tilde{\mathfrak{A}}, M, \sigma)$ admissible sequence $\left(x_{1}^{*}, \ldots, x_{j}^{*}\right)$ with $\max \operatorname{supp}\left(x_{j}\right)>\min \operatorname{supp} x$ and any $x^{*} \in \bigcup_{t \geq \sigma\left(x_{1}^{*}, \ldots, x_{j}^{*}\right)} A_{t}$ we have $\left|x^{*}(x)\right|<1+\varepsilon$. This last condition says that $x$ is a "good $\ell_{\infty}^{k}$-average" but for $k^{\prime} \gg k$ (where " $k^{\prime} \gg k$ " depends on min supp $x$ ) $x$ is a "bad $\ell_{\infty}^{k^{\prime}}$-average." We will call such a vector $x$ a special $\ell_{\infty}^{k}$-average of constant $1+\varepsilon$.

If $c_{0}$ is not block finitely representable in $E$, then for some $1 \leq q<\infty, \ell_{q}$ is block finitely representable in $E$. In this case we will be able to find a sequence $\left(z_{k}\right)$ in $y$ consisting of increasing $\ell_{q}$-averages. Furthermore, $\left(z_{k}\right)$ satisfies the assumptions of Lemma $2.2(\mathrm{~b})$ with $(\mathcal{D}, M, \delta)$ replaced by $(\mathcal{B}, M, \sigma)$ as well as by $(\tilde{\mathfrak{A}}, M, \sigma)$. Applying Lemma 2.2(b) will give us that by replacing $p$ by $q$ and $\left(y_{k}\right)$ by $\left(z_{k}\right)$ we find ourselves in the first case.

Step 3. Now we consider a spreading model of a sequence $\left(y_{n}\right)$ consisting of special $\ell_{\infty}^{k_{n}}$-averages of constant $\left(1+\varepsilon_{n}\right)$, where $k_{n} \uparrow \infty$ and $\varepsilon_{n} \downarrow 0$. Once again we have to distinguish between two cases.

Case 1. Up to passing to a subsequence we find a $C>0$ so that

$$
\varlimsup_{n_{1} \rightarrow \infty} \varlimsup_{n_{2} \rightarrow \infty} \ldots \varlimsup_{n_{m} \rightarrow \infty} \frac{f(m)}{m}\left\|\sum_{i=1}^{m} y_{n_{i}}\right\| \leq C,
$$

for all $m \in \mathbb{N}$.

Case 2. Up to passing to a subsequence of $\left(y_{n}\right)$ we find $c_{k} \downarrow 0$ and $m_{k} \uparrow \infty$ in $\mathbb{N}$ so that

$$
\varlimsup_{n_{1} \rightarrow \infty} \ldots \varlimsup_{n_{m_{k}} \rightarrow \infty} c_{k} \frac{f\left(m_{k}\right)}{m_{k}}\left\|\sum_{s=1}^{m_{k}} y_{n_{s}}\right\|=1 .
$$

In the second case we let $\left(z_{n}\right)$ be a block sequence of the form

$$
z_{n}=c_{n} \frac{f\left(m_{n}\right)}{m_{n}} \sum_{s=1}^{m_{n}} y_{k(n, s)}
$$

and observe that $\left(z_{n}\right)$ satisfies the conditions of Lemma $2.2(\mathrm{~b})$ for $\mathcal{D}=\tilde{\mathfrak{A}}$ as well as for $\mathcal{D}=\mathfrak{B}$, and deduce that $c_{0}$ is a spreading model of a subsequence of $\left(z_{n}\right)$. Taking a block sequence $\left(\tilde{y}_{n}\right)$ of the form

$$
\tilde{y}_{n}=\sum_{i=1}^{k_{n}} z_{m(n, i)}
$$

with $k_{n} \uparrow \infty$ we will observe that $\left(\tilde{y}_{n}\right)$ satisfies the hypothesis of Case 1 . Thus we can assume Case 1 to be satisfied.

In that case we will show that choosing $C(\bar{y})=C$ and letting $\varepsilon>0$ and taking $m_{0}$ sufficiently large we can choose for any $k \in \mathbb{N}, \delta>0$ and $m \in \operatorname{Im}(\delta)$, with $m \geq m_{0}$ and $\frac{f(m)}{m}<\frac{\delta}{8}$, a vector $y \in Y$ to be of the form

$$
y=\frac{f(m)}{m} \sum_{i=1}^{m} y_{n_{i}},
$$

in order to satisfy the claim of Lemma 3.4. 
We begin with an easy but important result.

Lemma 4.1. Let $\left(y_{i}\right)$ be a block basis of $\left(e_{i}\right)$ in $X$. If $c_{0}$ is block finitely represented in $\left(y_{i}\right)$, then so is $\ell_{1}$.

Proof. Given $n$ fixed we may choose a normalized block basis $\left(x_{i}\right)_{i=1}^{n}$ of $\left(y_{i}\right)$ along with functionals $x_{i}^{*} \in A_{m_{i}}$ so that

i) $x_{1}^{*}<x_{2}^{*}<\cdots<x_{n}^{*} ; x_{i}^{*}\left(x_{i}\right)>1 / 3$ and $x_{i}^{*}\left(x_{j}\right)=0$ for $1 \leq i \neq j \leq n$;

ii) for all $1 \leq k_{1}<k_{2}<\cdots<k_{\ell} \leq n$ and all choices of sign \pm

$$
\left( \pm x_{k_{1}}^{*}, \pm x_{k_{2}}^{*}, \ldots, \pm x_{k_{\ell}}^{*}\right) \text { is }(\tilde{\mathfrak{A}}, M, \sigma) \text {-admissible. }
$$

Indeed each $x_{i}$ will be a $(1+\varepsilon)-\ell_{\infty}^{m_{i}}$ normalized average for suitable $m_{i}$ and small $\varepsilon$. Thus if $\left(a_{i}\right)_{1}^{\ell} \subseteq \mathbb{R}$ and $1 \leq k_{1}<\cdots<k_{\ell} \leq n$ and $\varepsilon_{i}=\operatorname{sign} a_{i}$ we have $x^{*}=\frac{1}{f(\ell)} \sum_{i=1}^{\ell} \varepsilon_{i} x_{k_{i}}^{*} \in B_{\ell}$ and

$$
\left\|\sum_{1}^{\ell} a_{i} x_{k_{i}}\right\| \geq x^{*}\left(\sum_{i=1}^{\ell} a_{i} x_{k_{i}}\right) \geq \frac{1}{3 f(\ell)} \sum_{i=1}^{\ell}\left|a_{i}\right| .
$$

From James' proof that $\ell_{1}$ is not distortable we obtain that $\ell_{1}$ is block finitely represented in $\left(y_{i}\right)[\mathrm{J}]$.

From Lemma 4.1 and Krivine's theorem $([\mathrm{K}],[\mathrm{L}])$ we have

Corollary 4.2. If $\left(y_{i}\right)$ is a block basis of $\left(e_{i}\right)$, then there exists $p \in[1, \infty)$ so that $\ell_{p}$ is block finitely representable in $\left(y_{i}\right)$.

Lemma 4.3. Let $1 \leq p<\infty, 0<\varepsilon<1$ and $\ell \in \mathbb{N}$. Let $\left(y_{i}\right)$ be a block basis of $\left(e_{i}\right)$ and let $k_{1}<\cdots<k_{\ell}$ satisfy:

For $1 \leq i \leq \ell, y_{i}$ is an $\ell_{p}^{k_{i}}$-average with constant $1+\varepsilon$.

$$
f\left(\frac{k_{1}^{1 / p^{2}} \varepsilon^{2}}{10 \ell}\right) \geq \frac{2 \ell\left(1+2 C_{p}\right)}{\varepsilon},
$$

and for $i=1,2, \ldots, \ell-1$,

$$
\frac{\varepsilon}{2} f\left(k_{i+1}^{1 / p}\right) \geq \sum_{s=1}^{i}\left|\operatorname{supp} y_{i}\right| \text {. }
$$

Then for $\left(\alpha_{i}\right)_{1}^{\ell} \subseteq[-1,1]^{\ell}, y=\sum_{i=1}^{\ell} \alpha_{i} y_{i}$ and $m \geq 2$,

$$
\begin{gathered}
\text { if } x^{*}=\frac{1}{f(m)} \sum_{j=1}^{m} x_{j}^{*} \in B_{m}, \\
\left|x^{*}(y)\right| \leq\left\{\begin{array}{lc}
\frac{1}{f(m)}\left(\max _{j \leq m}\left|x_{j}^{*}(y)\right|+\varepsilon\right), & m \leq \frac{\varepsilon^{2} k_{1}^{1 / p^{2}}}{10 \ell}, \\
\max _{i \leq \ell}\left|\alpha_{i}\right|(1+\varepsilon)+\varepsilon, & m>\frac{\varepsilon^{2} k_{1}^{1 / p^{2}}}{10 \ell}
\end{array}\right. \\
\leq\left\{\begin{array}{lc}
\frac{1}{f(m)}(\|y\|+\varepsilon), & m \leq \frac{\varepsilon^{2} k_{1}^{1 / p^{2}}}{10 \ell}, \\
\max _{i \leq \ell}\left|\alpha_{i}\right|(1+\varepsilon)+\varepsilon, & m>\frac{\varepsilon^{2} k_{1}^{1 / p^{2}}}{10 \ell} .
\end{array}\right.
\end{gathered}
$$


(4.3.4) For $x^{*}=\frac{1}{m} \sum_{i=1}^{m} x_{i}^{*} \in A_{m}$,

$$
\begin{aligned}
\left|x^{*}(y)\right| \leq \max _{s \leq \ell}\left|\alpha_{s}\right|\left[k_{1}^{-1 / p}+4\left(\frac{\min (\ell, m)}{m}\right)^{1 / p}\right] \\
+\frac{1}{m} \max \left\{\sum_{j=1}^{\ell^{\prime}}\left\|\sum_{i \in E_{j}} \alpha_{i} y_{i}\right\|: \ell^{\prime} \leq \min (\ell, m) E_{1}<\cdots<\right.
\end{aligned}
$$

$$
\left.E_{\ell^{\prime}} \text { are intervals in }\{1, \ldots, \ell\}\right\} \text {. }
$$

Proof. Let $x^{*}=\frac{1}{f(m)} \sum_{i=1}^{m} x_{i}^{*} \in B_{m}$ where $x_{i}^{*}=\frac{1}{m_{i}} \sum_{j=1}^{m_{i}} x^{*}(i, j) \in A_{m_{i}}$.

Case $1 . m \leq \varepsilon^{2} k_{1}^{1 / p^{2}} / 10 \ell$.

Let $j_{0} \in\{1,2, \ldots, m+1\}$ be maximal so that $\sum_{i=1}^{j_{0}-1}\left|\operatorname{supp} x_{j}^{*}\right| \leq \varepsilon k_{1}^{1 / p} / 2$. Thus if $j_{0}<m$ from $\left(\sigma_{2}\right)$ we have $m_{j_{0}+1}>\sum_{i=1}^{j_{0}}\left|\operatorname{supp} x_{j}^{*}\right|>\frac{\varepsilon k_{1}^{1 / p}}{2}$. Thus by Corollary 3.6,

$$
\begin{aligned}
\sum_{j=j_{0}+1}^{m}\left|x_{j}^{*}(y)\right| & \leq \sum_{i=1}^{\ell} \sum_{j=j_{0}+1}^{m}\left|x_{j}^{*}\left(y_{i}\right)\right| \\
& \leq \ell m\left[k_{1}^{-1 / p}+2 m_{j_{0}+1}^{-1 / p}\right] \\
& \leq \ell m\left[k_{1}^{-1 / p}+2 \cdot 2^{1 / p} \varepsilon^{-1 / p} k_{1}^{-1 / p^{2}}\right] \\
& \leq 5 \ell m \varepsilon^{-1} k_{1}^{-1 / p^{2}}<\varepsilon / 2 .
\end{aligned}
$$

Also,

$$
\begin{aligned}
\sum_{j=1}^{j_{0}-1}\left|x_{j}^{*}(y)\right| & \leq \sum_{j=1}^{j_{0}-1}\left|\operatorname{supp} x_{j}^{*}\right|\|y\|_{\infty} \\
& \leq k_{1}^{-1 / p} \sum_{j=1}^{j_{0}-1}\left|\operatorname{supp} x_{j}^{*}\right|<\varepsilon / 2
\end{aligned}
$$

by our choice of $j_{0}$ and the fact that

$$
\|y\|_{\infty} \leq \max _{i}\left\|y_{i}\right\|_{\infty} \leq \max _{i} k_{i}^{-1 / p}=k_{1}^{-1 / p} .
$$

Thus

$$
\left|x^{*}(y)\right| \leq \frac{1}{f(m)}\left|x_{j_{0}}^{*}(y)\right|+\varepsilon .
$$

Case 2. $m>\varepsilon^{2} k_{1}^{1 / p^{2}} / 10 \ell$.

Choose $i_{0} \in\{1, \ldots, \ell+1\}$ maximal so that

$$
\sum_{i=1}^{i_{0}-1}\left|\operatorname{supp} y_{i}\right|<\frac{\varepsilon f(m)}{2} \text {. }
$$

Then

$$
\left|x^{*}\left(\sum_{i=1}^{i_{0}-1} \alpha_{i} y_{i}\right)\right|<\frac{\varepsilon}{2} .
$$


Also, by (4.3.2) if $i_{0}<\ell$,

$$
\frac{\varepsilon f(m)}{2} \leq \sum_{i=1}^{i_{0}}\left|\operatorname{supp} y_{i}\right| \leq \frac{\varepsilon}{2} f\left(k_{i_{0}+1}^{1 / p}\right)
$$

which yields $m \leq k_{i_{0}+1}^{1 / p}$. If $i_{0}<i \leq \ell$, we have

$$
\left|x^{*}\left(y_{i}\right)\right| \leq \frac{1}{f(m)} \sum_{j=1}^{m}\left|x_{j}^{*}\left(y_{i}\right)\right| \leq \frac{1}{f(m)} \sum_{j=1}^{m}\left(k_{i}^{-1 / p}+2 m_{j}^{-1 / p}\right)
$$

by Corollary 3.6 and in turn by $\left(\sigma_{3}\right)$ this is

$$
\begin{aligned}
& \leq \frac{1}{f(m)}\left[m k_{i_{0}+1}^{-1 / p}+2 C_{p}\right] \leq \frac{1}{f(m)}\left[1+2 C_{p}\right] \\
& \leq \frac{1}{f\left(\varepsilon^{2} k_{1}^{1 / p^{2}} / 10 \ell\right)}\left[1+2 C_{p}\right] \leq \frac{\varepsilon}{2 \ell}
\end{aligned}
$$

where the last inequality follows from (4.3.2).

Thus $\left|x^{*}\left(\sum_{i=i_{0}+1}^{\ell} \alpha_{i} y_{i}\right)\right| \leq \varepsilon / 2$. We obtain $\left|x^{*}(y)\right| \leq \varepsilon+\left|x^{*}\left(\alpha_{i_{0}} y_{i_{0}}\right)\right|$ which completes the proof of (4.3.3), since $\left\|y_{i_{0}}\right\|<1+\varepsilon$.

Let $x^{*}=\frac{1}{m} \sum_{1}^{m} x_{i}^{*} \in A_{m}$ and let $1=n_{1}<n_{2}<\cdots<n_{\ell+1}$ so that $\operatorname{supp} y_{i} \subseteq$ $\left[n_{i}, n_{i+1}\right)$ for $1 \leq i \leq \ell$ and $n_{\ell+1}>\max \operatorname{supp} x_{m}^{*}$. For $1 \leq i \leq \ell$ define $I_{i}=\{j$ : $\left.\operatorname{supp} x_{j}^{*} \subseteq\left[n_{i}, n_{i+1}\right)\right\}$ and $m_{i}=\left|I_{i}\right|$. Note that $\sum_{i=1}^{\ell} \frac{m_{i}}{m} \leq 1$. If $I_{i} \neq \emptyset$, then $\frac{1}{m_{i}} \sum_{j \in I_{i}} x_{j}^{*} \in A_{m_{i}}$ and so by Corollary 3.6,

$$
\begin{aligned}
\left|\sum_{j \in I_{i}} x_{j}^{*}\left(\alpha_{i} y_{i}\right)\right| & =m_{i} \frac{1}{m_{i}}\left|\sum_{j \in I_{i}} x_{j}^{*}\left(y_{i}\right)\right|\left|\alpha_{i}\right| \\
& \leq m_{i}\left[k_{i}^{-1 / p}+2 m_{i}^{-1 / p}\right]\left|\alpha_{i}\right| .
\end{aligned}
$$

Hence if " $\sum_{i=1}^{\ell}$ " denotes " $\sum_{\substack{i=1 \\ I_{i} \neq \emptyset}}^{\ell}$, then

$$
\begin{aligned}
\frac{1}{m} \sum_{i=1}^{\ell}\left|\sum_{j \in I_{i}} x_{j}^{*}\left(\alpha_{i} y_{i}\right)\right| & \leq \sum_{i=1}^{\ell} \frac{m_{i}}{m} k_{i}^{-1 / p}\left|\alpha_{i}\right|+2 \sum_{i=1}^{\ell^{\prime}} \frac{m_{i}}{m} m_{i}^{-1 / p}\left|\alpha_{i}\right| \\
& \leq \max _{s \leq \ell}\left|\alpha_{s}\right|\left[k_{1}^{-1 / p}+2 \sum_{i=1}^{\ell} \frac{m_{i}}{m} m_{i}^{-1 / p}\right] .
\end{aligned}
$$

If $m \leq \ell$, then we use the estimate,

$$
\sum_{i=1}^{\ell} \frac{m_{i}}{m} m_{i}^{-1 / p} \leq \max _{\substack{i \leq \ell \\ I_{i} \neq \emptyset}} m_{i}^{-1 / p} \leq 1 .
$$

If $m>\ell$, then by Hölder's inequality,

$$
\begin{aligned}
\frac{1}{m} \sum_{i=1}^{\ell} m_{i}^{1-1 / p} & \leq \frac{1}{m}\left(\sum_{i=1}^{\ell}{ }^{\prime} 1^{p}\right)^{1 / p}\left(\sum_{i=1}^{\ell} m_{i}\right)^{1-1 / p} \\
& \leq \frac{\ell^{1 / p}}{m} m^{1-1 / p}=\left(\frac{\ell}{m}\right)^{1 / p} .
\end{aligned}
$$


Thus

$$
\frac{1}{m} \sum_{i=1}^{\ell}\left|\sum_{j \in I_{i}} x_{j}^{*}\left(\alpha_{i} y_{i}\right)\right| \leq \max _{s \leq \ell}\left|\alpha_{s}\right|\left[k_{1}^{-1 / p}+2\left(\frac{\min (\ell, m)}{m}\right)^{1 / p}\right] .
$$

Let $I_{0}=\{1,2, \ldots, m\} \backslash \bigcup_{i=1}^{\ell} I_{i}$ and $\ell^{\prime}=\left|I_{0}\right| \leq \min (\ell, m)$. Then for an appropriate choice of $k_{1}<\cdots<k_{\ell^{\prime}}$ and intervals $E_{1}<\cdots<E_{m^{\prime}} \subseteq\{1, \ldots, \ell\}$,

$$
\sum_{j \in I_{0}}\left|x_{j}^{*}(y)\right| \leq \sum_{j=1}^{\ell^{\prime}}\left|\alpha_{k_{j}}\right|\left\|y_{k_{j}}\right\|+\sum_{j=1}^{\ell^{\prime}}\left\|\sum_{i \in E_{j}} \alpha_{i} y_{i}\right\| \text {. }
$$

Since

$$
\sum_{j=1}^{\ell^{\prime}}\left|\alpha_{k_{j}}\right|\left\|y_{k_{j}}\right\| \leq \min (\ell, m) \max _{s \leq \ell}\left|\alpha_{s}\right|(1+\varepsilon)
$$

(4.3.4) follows from these estimates using that

$$
\left|x^{*}(y)\right| \leq \frac{1}{m} \sum_{i=1}^{\ell^{\prime}}\left|\sum_{j \in I_{i}} x^{*}\left(\alpha_{i} y_{i}\right)\right|+\frac{1}{m} \sum_{j \in I_{0}}\left|x_{j}^{*}(y)\right| .
$$

Remark 4.4. By Corollary 4.2 for every block basis $\left(x_{i}\right)$ of $\left(e_{i}\right)$ there exists $1 \leq p<$ $\infty$ so that for all $\varepsilon>0$ and $\ell \in \mathbb{N}$ there exists a block basis $\left(y_{i}\right)_{i=1}^{\ell}$ of $\left(x_{i}\right)$ satisfying (4.3.1) and (4.3.2).

Lemma 4.5. Let $\left(x_{i}\right)$ be a block basis of $\left(e_{i}\right), \varepsilon>0$ and $k \in \mathbb{N}$. There exists $x \in \operatorname{span}\left(x_{i}\right)$ so that

(4.5.1) $x$ is an $\ell_{\infty}^{k}$-average with constant $1+\varepsilon$, and

(4.5.2) if $\left(x_{1}^{*}, \ldots, x_{j}^{*}\right)$ is $(\tilde{\mathfrak{A}}, M, \sigma)$-admissible with $\max \operatorname{supp} x_{j}^{*} \geq \min \operatorname{supp} x$, then

$$
\left|x^{*}(x)\right|<\varepsilon \text { for all } x^{*} \in \bigcup_{t \geq \sigma\left(x_{1}^{*}, \ldots, x_{j}^{*}\right)} A_{t} .
$$

Proof. As in Remark 4.4 there exists $1 \leq p<\infty$ and a block basis $\left(y_{i}\right)$ of $\left(x_{i}\right)$ and a subsequence $\left(k_{i}\right)$ of $\mathbb{N}$ satisfying for $\varepsilon_{i} \equiv \varepsilon / 2^{i}$,

(4.5.3) for every $i, y_{i}$ is an $\ell_{p}^{k_{i}}$-average with constant $1+\varepsilon_{i}$;

(4.5.4) for $\ell \in \mathbb{N}$ and $\ell \leq n_{1}<\cdots<n_{\ell}$ :

a) $f\left(k_{n_{1}}^{1 / p^{2}} \varepsilon_{\ell}^{2} / 10 \ell\right)>2 \ell\left(1+2 C_{p}\right) / \varepsilon_{\ell}$

b) $\frac{\varepsilon_{\ell}}{2} f\left(k_{n_{i+1}}^{1 / p}\right) \geq \sum_{s=1}^{i}\left|\operatorname{supp} y_{n_{s}}\right|$ for $1 \leq i<\ell$,

c) $\left(y_{i}\right)$ has a spreading model $\left(\tilde{y}_{i}\right)$ satisfying for $\left(\alpha_{i}\right)_{1}^{\ell} \in[-1,1]^{\ell}$,

$$
\left|\left\|\sum_{i=1}^{\ell} \alpha_{i} y_{n_{i}}\right\|-\left\|\sum_{i=1}^{\ell} \alpha_{i} \tilde{y}_{i}\right\|\right|<\varepsilon_{\ell} .
$$

Indeed we first choose a sequence $\left(y_{i}\right)$ satisfying a) and b) for all subsequences and then pass to a subsequence satisfying $\mathrm{c}$ ).

Case 1. $c_{0}$ is block finitely representable in $\left(\tilde{y}_{i}\right)$.

Using c) we can thus find $N$ so that if $N<n_{1}<\cdots<n_{N}$, then there exists a normalized block basis $\left(w_{i}\right)_{1}^{k}$ of $\left(y_{n_{i}}\right)_{1}^{N}$ which is $(1+\varepsilon)$-equivalent to the unit vector basis of $\ell_{\infty}^{k}$. Thus $x=\sum_{1}^{k} w_{i}$ is an $\ell_{\infty}^{k}$-average with constant $1+\varepsilon$. Now we do this 
choosing $n_{1}$ so large that $k_{n_{1}}^{-1 / p}<\varepsilon / 6$ and if $\left(x_{1}^{*}, \ldots, x_{j}^{*}\right)$ is $(\tilde{\mathfrak{A}}, M, \sigma)$-admissible with $\max \operatorname{supp} x_{j}^{*} \geq \min \operatorname{supp} y_{n_{1}}$, then $\sigma\left(x_{1}^{*}, \ldots, x_{j}^{*}\right)>(24 / \varepsilon)^{p} N$.

We can write $x=\sum_{1}^{N} \alpha_{i} y_{n_{i}}$ for some $\left(\alpha_{i}\right)_{1}^{N} \subseteq[-2,2]^{\mathbb{N}}$. Let $m \geq(24 / \varepsilon)^{p} N$ and let $x^{*} \in A_{m}$. From (4.3.4) we obtain (we may assume $\varepsilon / 24<1$ ),

$$
\left|x^{*}(x)\right| \leq 2\left[k_{n_{1}}^{-1 / p}+4\left(\frac{N}{m}\right)^{1 / p}\right]+\frac{2 N}{m}<2\left[\frac{\varepsilon}{6}+\frac{\varepsilon}{6}\right]+\frac{2 N}{m}<\varepsilon .
$$

If Case 1 fails to hold, then by Krivine's theorem ([K], $[\mathrm{L}]$ ) we have

Case $2 . \ell_{q}$ is block finitely represented in $\left(\tilde{y}_{i}\right)$ for some $1 \leq q<\infty$.

In this case we produce in $\left(y_{i}\right)$ a block basis $\left(z_{i}\right)$ of $\ell_{q}^{k_{i}^{\prime}}$-averages with constant $1+\varepsilon_{i}$ satisfying $k_{i}^{\prime} \uparrow \infty$ and for all $\ell \leq n_{1}<\cdots<n_{\ell}$ :

a) $f\left(k_{n_{1}}^{\prime}{ }^{1 / q^{2}} \varepsilon_{\ell}^{2} / 10 \ell\right)>2 \ell\left(1+2 C_{q}\right) / \varepsilon_{\ell}$,

b) $\frac{\varepsilon_{\ell}}{2} f\left(k_{n_{i+1}}^{\prime}{ }^{1 / q}\right) \geq \sum_{s=1}^{i}\left|\operatorname{supp} z_{n_{s}}\right|$ for $1 \leq i<\ell$ where $\operatorname{supp}\left(z_{n_{s}}\right)$ is w.r.t. $\left(y_{t}\right)$ and

$$
z_{i}=\sum_{j=1}^{N_{i}} \alpha(i, j) y_{n(i, j)}
$$

for some $N_{i}<n(i, 1)<\cdots<n\left(i, N_{i}\right)$. The latter yields by (4.3.3) that for $i, m \in \mathbb{N}$ and $x^{*} \in B_{m}$,

$$
\left|x^{*}\left(z_{i}\right)\right| \leq \frac{1}{f(m)}\left(\left\|z_{i}\right\|+\varepsilon_{i}\right)+\varepsilon_{i}+\left(1+\varepsilon_{i}\right) \max \left\{|\alpha(i, j)|: 1 \leq j \leq N_{i}\right\} .
$$

Since $z_{i}$ is an $\ell_{q}^{k_{i}^{\prime}}$-average in $\left(y_{s}\right)$ with $k_{i}^{\prime} \rightarrow \infty$ and $q<\infty$, it follows that

$$
\lim _{n \rightarrow \infty} \sup \left\{\left|x^{*}\left(z_{i}\right)\right|: i, m \geq n, x^{*} \in B_{m}\right\}=0 .
$$

Thus the hypothesis of Lemma $2.2 \mathrm{~b}$ ) is satisfied with $\mathcal{D}, M, \sigma$ replaced by $\mathfrak{B}, M, \sigma$ and $y_{i}$ replaced by $\frac{z_{i}}{\left\|z_{i}\right\|} \rightarrow 1$. Hence for all $\left(\alpha_{i}\right)_{1}^{\ell} \subseteq \mathbb{R}$,

$$
\varlimsup_{n_{1} \rightarrow \infty} \ldots \varlimsup_{n_{\ell} \rightarrow \infty}\left\|\sum_{i=1}^{\ell} \alpha_{i} z_{n_{i}}\right\| \leq \max \left\{\left\|\left(\alpha_{i}\right)\right\|_{\infty}, \max _{j \leq \ell} \varlimsup_{n_{j} \rightarrow \infty} \ldots \varlimsup_{n_{\ell} \rightarrow \infty}\left\|\sum_{i=j}^{\ell} \alpha_{i} z_{n_{i}}\right\|_{B}\right\} .
$$

But by (4.3.3), which applies, due to $\left.\mathrm{a}^{\prime}\right)$ and $\left.\mathrm{b}^{\prime}\right)$,

$$
\varlimsup_{n_{1} \rightarrow \infty} \ldots \varlimsup_{n_{\ell} \rightarrow \infty}\left\|\sum_{i=1}^{\ell} \alpha_{i} z_{n_{i}}\right\|_{B} \leq \max \left\{\left\|\left(\alpha_{i}\right)\right\|_{\infty}, \frac{1}{f(2)} \varlimsup_{n_{1} \rightarrow \infty} \ldots \varlimsup_{n_{\ell} \rightarrow \infty}\left\|\sum_{i=1}^{\ell} \alpha_{i} z_{n_{i}}\right\|\right\}
$$

which together with the above inequality implies

$$
\varlimsup_{n_{1} \rightarrow \infty} \ldots \varlimsup_{n_{\ell} \rightarrow \infty}\left\|\sum_{i=1}^{\ell} \alpha_{i} z_{n_{i}}\right\|=\left\|\left(\alpha_{i}\right)\right\|_{\infty} .
$$

The lemma follows by this and (4.3.4) if we set $x=\sum_{1}^{k} z_{n_{i}} /\left\|z_{n_{i}}\right\|$ for a suitable choice of $n_{1}<\cdots<n_{k}$. 
Proof of the Main Lemma 3.4. By virtue of Lemma 4.5 we can choose a block sequence $\left(y_{i}\right)$ in $Y$ along with sequences $\varepsilon_{i} \downarrow 0$ with $\varepsilon_{1}<1 / 2$ and a subsequence $\left(k_{i}\right)$ of $\mathbb{N}$ so that conditions (1) and (2) hold for all $i \in \mathbb{N}$.

(1) a) $y_{i}$ is an $\ell_{\infty}^{k_{i}}$-average with constant $\left(1+\varepsilon_{i}\right)$.

b) If $\left(x_{1}^{*}, \ldots, x_{j}^{*}\right)$ is $(\tilde{\mathfrak{A}}, M, \sigma)$-admissible with $\max \operatorname{supp} x_{j}^{*} \geq \min \operatorname{supp} y_{i}$, then

$$
\left|x^{*}\left(y_{i}\right)\right|<\varepsilon_{i} \text { for all } x^{*} \in \bigcup_{m \geq \sigma\left(x_{1}^{*}, \ldots, x_{j}^{*}\right)} A_{m} .
$$

c) There exists $\left(y_{i}^{*}\right) \subseteq B\left(X^{*}\right) \cap A$ with $\operatorname{supp} y_{i}^{*} \subseteq\left[\min \operatorname{supp} y_{i}\right.$, max supp $\left.y_{i}\right]$, $y_{i}^{*}\left(y_{i}\right)>1 / 3$ and $y_{i+1}^{*} \in A_{k_{i+1}}$ where $k_{i+1} \geq \sigma\left(y_{1}^{*}, \ldots, y_{i}^{*}\right)$

(2) $\frac{1}{\varepsilon_{i}} \max \operatorname{supp} y_{i}<f\left(\frac{\varepsilon_{i}}{\varepsilon_{i+1}}\right)$.

Note that any subsequence of $\left(y_{i}, \varepsilon_{i}, k_{i}\right)_{\mathbb{N}}$ also satisfies conditions (1) and (2) (for condition c) this uses $\left.\left(\sigma_{1}\right)\right)$.

Let $m \in \mathbb{N}, m<n_{1}<\cdots<n_{m}$, set $x_{i}=y_{n_{i}}$ for $i \leq m$ and $x=\sum_{1}^{m} x_{i}$.

We first obtain estimates for $\|x\|_{A_{\ell}}$ and $\|x\|_{B_{\ell}}$ for $\ell \geq 2$. From Lemma 3.5 and the fact that $\left\|x_{i}\right\| \leq 1+\varepsilon_{m}$ for $i \leq m$ we have

$$
\|x\|_{A_{\ell}} \leq(1+\varepsilon)\left(1+\frac{m}{\ell}\right)<2 \frac{\ell+m}{\ell} .
$$

From (1) b) and (2) and the fact that $\varepsilon_{i} \downarrow$ it is easy to check that Lemma $2.2 \mathrm{a}$ ) applies for $\varepsilon=\varepsilon_{m}$ and $\mathcal{D}=\tilde{\mathfrak{A}}, k=m$ (and $y_{i}$ in Lemma 2.2 replaced by $\frac{x_{i}}{1+\varepsilon_{m}}$ ). We obtain from the second estimate in (2.2.2) that

$$
\begin{aligned}
\|x\|_{B_{\ell}} \leq \sup _{\ell^{\prime} \geq \ell} & \frac{\|x\|_{A_{\ell^{\prime}}}}{f(\ell)}+\frac{\min (\ell, m)}{f(\ell)} \sup _{\ell^{\prime} \geq m}\|x\|_{A_{\ell^{\prime}}} \\
& +\left(1+\varepsilon_{m}\right)\left[1+\frac{1}{f(\ell)}+2 \varepsilon_{m}+\frac{2}{f(\ell)}\right] .
\end{aligned}
$$

We have used that $x_{1}^{*} \in A_{t}$ for $t \geq M_{\ell} \geq \ell$ if $\left(x_{1}^{*}, \ldots, x_{j}^{*}\right)$ is $(\tilde{\mathfrak{A}}, M, \sigma)$-admissible to obtain the first term.

Thus since $\sup _{\ell^{\prime} \geq m}\|x\|_{A_{\ell^{\prime}}}<4$ by (3) we obtain, using $\varepsilon_{m}<\varepsilon_{1}<\frac{1}{2}$,

$$
\|x\|_{B_{\ell}} \leq \sup _{\ell^{\prime} \geq \ell} \frac{\|x\|_{A_{\ell^{\prime}}}}{f(\ell)}+9 \frac{\min (\ell, m)}{f(\ell)}+3 .
$$

Let $\left(\tilde{y}_{i}\right)$ be a spreading model of a subsequence of $\left(y_{i}\right)$. It may be that for some constant $C$ we have that $C(m) \equiv \frac{f(m)}{m}\left\|\sum_{i=1}^{m} \tilde{y}_{i}\right\|<C$ for all $m$. If so, then by passing to a subsequence of $\left(y_{i}\right)$ we may assume that we have

Case 1. For all $m<n_{1}<\cdots<n_{m}$,

$$
\frac{f(m)}{m}\left\|\sum_{1}^{m} y_{n_{i}}\right\| \leq C
$$

In the remaining case we have $\varlimsup_{m} C(m)=\infty$. Select a subsequence $C\left(m_{n}\right) \uparrow \infty$ with for all $n, C(i)<C\left(m_{n}\right)$ if $i<m_{n}$. Thus

$$
\frac{1}{C\left(m_{n}\right)} \frac{f\left(m_{n}\right)}{m_{n}}\left\|\sum_{j=1}^{m_{n}} \tilde{y}_{j}\right\|=1
$$


and if $m^{\prime}<m_{n}$, then

$$
\frac{1}{C\left(m_{n}\right)} \frac{f\left(m^{\prime}\right)}{m^{\prime}}\left\|\sum_{1}^{m^{\prime}} \tilde{y}_{j}\right\|<1 .
$$

Set $c_{n} \equiv C\left(m_{n}\right)^{-1}$. Thus $c_{n} \downarrow 0$. Hence if Case 1 fails to hold we have

Case 2. There exists a block basis $\left(z_{n}\right)$ of $\left(y_{i}\right)$ where

$$
z_{n}=c_{n} \frac{f\left(m_{n}\right)}{m_{n}} \sum_{s=1}^{m_{n}} y_{k(n, s)}
$$

for some $m_{n}<k(n, 1)<\cdots<k\left(n, m_{n}\right)$ and $\left\|z_{n}\right\|$ is chosen so that $\left|\left\|z_{n}\right\|-1\right|<\varepsilon_{n}$ and if $F \varsubsetneqq\left\{1, \ldots, m_{n}\right\}$, then

$$
\left\|\sum_{s \in F} y_{k(n, s)}\right\|<\frac{|F|}{c_{n} f(|F|)} .
$$

We return now to Case 1 and complete the proof of Lemma 3.4 in this situation. Let $\varepsilon>0$. Choose $m_{0}$ so that (as usual $\bar{m}$ and $\overline{\bar{m}}$ are the predecessor and successor of $m$ in $\operatorname{Im}(\sigma))$

$$
\begin{aligned}
& \frac{C}{f\left(m_{0}\right)}+\frac{f\left(m_{0}\right)}{m_{0}}<\frac{\varepsilon}{4}, \\
& \sup _{\ell, m \geq m_{0}} C \frac{f(m)}{\ell f\left(\frac{m}{\min (\ell, m)}\right)}<\frac{\varepsilon}{2}, \text { and } \\
& \sup \left\{\frac{f(m) \min (\ell, m)}{m f(\ell)}: \quad \begin{array}{l}
\left.m \in \operatorname{Im}(\sigma), m \geq m_{0} \text { and }, \bar{m}\right] \cup[\bar{m}, \infty) \\
\ell \in\left[m_{0}, \infty\right.
\end{array}\right\}<\frac{\varepsilon}{36},
\end{aligned}
$$

where $\left(\sigma_{4}\right)$ is used to get $(8)$.

To verify ( $*$ ) of Lemma 3.4 we let $k \in \mathbb{N}, \delta>0$ and $m \in \operatorname{Im}(\sigma)$ with $m \geq m_{0}$ and $\frac{f(m)}{m}<\frac{\delta}{8}$. Choose $n_{0}>\max \left\{k, M_{m}\right\}$ so that if $\left(x_{1}^{*}, \ldots, x_{j}^{*}\right)$ is $(\tilde{\mathfrak{A}}, M, \sigma)$-admissible with $\max \operatorname{supp} x_{j}^{*} \geq \min \operatorname{supp} y_{n_{0}}$, then $\sigma\left(x_{1}^{*}, \ldots, x_{j}^{*}\right) \geq m$ while if $\left(x_{1}^{*}, \ldots, x_{j}^{*}\right)$ were $(\mathfrak{B}, M, \sigma)$-admissible with $\max \operatorname{supp} x_{j}^{*} \geq \min \operatorname{supp} y_{n_{0}}$, then

$$
f\left(\sigma\left(x_{1}^{*}, \ldots, x_{j}^{*}\right)\right)>\frac{20 f(m)}{\delta} .
$$

Choose $n_{0}<n_{1}<\cdots<n_{m}$. Thus, by Case 1 ,

$$
\left\|\frac{f\left(m^{\prime}\right)}{m^{\prime}} \sum_{s \in F} y_{n_{s}}\right\| \leq C \text { for } F \subseteq\{1, \ldots, m\}, \quad|F|=m^{\prime} .
$$

Define $y=\frac{f(m)}{m} \sum_{i=1}^{m} y_{n_{i}}$. We have $\|y\| \leq C$ and $e_{k}<y$. Also by (1) c), $\left(\sigma_{1}\right)$ and the fact that $n_{0}>M_{m}$ there exists $y^{*} \in B_{m}$ with $e_{k}^{*}<y^{*}$ and

$$
y^{*}(y)>\frac{1}{f(m)} \frac{f(m)}{m} \sum_{i=1}^{m} \frac{1}{3}=\frac{1}{3} .
$$

We have verified a), b) of Lemma 3.4 and c) with constant $1 / 3$ rather than 1 . However this "weaker result" will formally imply the stated version. It remains to check conditions d) and e).

Condition d) i) follows from Lemma 3.5 and the choice of $m_{0}$. Indeed for $\ell \geq m_{0}$ 


$$
\begin{aligned}
\|y\|_{A_{\ell}} & \leq 2 \frac{f(m)}{m}+\frac{f(m)}{m} \frac{1}{\ell} \max \left\{\sum_{j=1}^{\ell^{\prime}} \frac{C m_{i}}{f\left(m_{i}\right)}: \ell^{\prime} \leq \min (\ell, m), m_{1}+\cdots+m_{\ell^{\prime}}=m\right\} \\
& \leq 2 \frac{f(m)}{m}+\frac{f(m)}{\ell m} \max _{\ell^{\prime} \leq \min (\ell, m)}\left\{\frac{C m}{f\left(\frac{m}{\ell^{\prime}}\right)}\right\} \\
& \leq 2 \frac{f(m)}{m}+\frac{C f(m)}{\ell} \frac{1}{f\left(\frac{m}{\min (\ell, m)}\right)}<\varepsilon .
\end{aligned}
$$

We have used the concavity of $x / f(x)$ along with (6), (7), (10), $\left\|y_{i}\right\|<2$ and the fact that $m \geq m_{0}$.

Let $\ell \in\left[m_{0}, \bar{m}\right] \cup[\overline{\bar{m}}, \infty)$. From $(3)$

$$
\begin{aligned}
\|y\|_{B_{\ell}} & \leq \frac{f(m)}{m f(\ell)} \sup _{\ell^{\prime} \geq \ell}\left\|\sum_{1}^{m} y_{n_{i}}\right\|_{A_{\ell^{\prime}}}+9 \frac{\min (\ell, m)}{f(\ell)} \frac{f(m)}{m}+\frac{3 f(m)}{m} \\
& \leq \frac{C}{f(\ell)}+9 \frac{f(m) \min (\ell, m)}{m f(\ell)}+\frac{3 f(m)}{m} \\
& <\varepsilon
\end{aligned}
$$

(using (10), (6), (8) and $m \geq m_{0}, \ell \geq m_{0}$ ). Thus d) ii) holds.

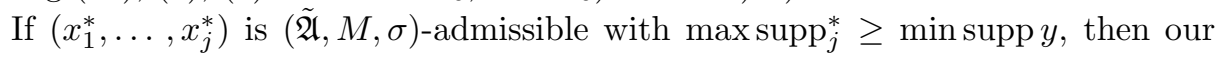
choice of $n_{0}$ implies that $\sigma\left(x_{1}^{*}, \ldots, x_{j}^{*}\right) \geq m$. Furthermore for $\ell \geq m$ from (3) we have

$$
\|y\|_{A_{\ell}} \leq \frac{f(m)}{m} 2 \frac{\ell+m}{\ell} \leq 4 \frac{f(m)}{m}<\delta .
$$

Thus (e) i) of Lemma 3.4 holds.

If $\left(y_{1}^{*}, \ldots, y_{j}^{*}\right)$ is $(\mathfrak{B}, M, \sigma)$-admissible with $\max \operatorname{supp} y_{j}^{*} \geq \min \operatorname{supp} y$, then for $\ell \geq \sigma\left(y_{1}^{*}, \ldots, y_{j}^{*}\right)$ it follows from (4), (9) and (11) that

$$
\|y\|_{B_{\ell}} \leq \frac{\delta}{f(\ell)}+9 \frac{f(m)}{f(\ell)}+3 \frac{f(m)}{m}<\delta
$$

Thus e) ii) holds.

Now let $\left(z_{n}\right)$ be as described in Case 2 above. For $\ell \geq 2$ by Lemma 3.5 and (5),

$$
\begin{aligned}
\left\|z_{n}\right\|_{A_{\ell}} \leq & \frac{2 c_{n} f\left(m_{n}\right)}{m_{n}} \\
& +\frac{c_{n} f\left(m_{n}\right)}{\ell m_{n}} \max \left\{\sum_{j=1}^{\ell^{\prime}} \frac{k_{j}}{c_{n} f\left(k_{j}\right)}: \ell^{\prime} \leq \min \left(\ell, m_{n}\right), k_{1}+\cdots+k_{\ell^{\prime}}=m_{n}\right\} \\
\leq & \frac{2 c_{n} f\left(m_{n}\right)}{m_{n}}+\frac{f\left(m_{n}\right)}{\ell m_{n}} \frac{m_{n}}{f\left(\frac{m_{n}}{\min \left(\ell, m_{n}\right)}\right)} \\
= & \frac{2 c_{n} f\left(m_{n}\right)}{m_{n}}+\frac{f\left(m_{n}\right)}{\ell f\left(\frac{m_{n}}{\min \left(\ell, m_{n}\right)}\right)} \rightarrow \frac{1}{\ell}
\end{aligned}
$$

as $n \rightarrow \infty$. 
Furthermore, from (12) we obtain,

$$
\lim _{i \rightarrow \infty} \sup \left\{\left\|z_{n}\right\|_{A_{\ell}}: n, \ell \geq i\right\}=0 .
$$

For $\ell \geq 2$ by (4)

$$
\left\|z_{n}\right\|_{B_{\ell}} \leq\left(1+\varepsilon_{n}\right) \frac{1}{f(\ell)}+9 c_{n} \frac{f\left(m_{n}\right)}{m_{n}} \frac{\min \left(\ell, m_{n}\right)}{f(\ell)}+3 c_{n} \frac{f\left(m_{n}\right)}{m_{n}},
$$

where we have used

$$
\left\|z_{n}\right\|_{A_{\ell}} \leq\left\|z_{n}\right\| \leq 1+\varepsilon_{n} .
$$

It follows that we have, using $c_{n} \rightarrow 0$,

$$
\lim _{i \rightarrow \infty} \sup \left\{\left\|z_{n}\right\|_{B_{\ell}}: \ell, n \geq i\right\}=0 .
$$

Hence Lemma 2.2 b) applies for $\mathcal{D}$ replaced by either $\tilde{\mathfrak{A}}$ or $\mathfrak{B}$ (we do not have $\left\|z_{n}\right\| \leq 1$ but rather $\left\|z_{n}\right\| \rightarrow 1$ which suffices $)$. For all $k$ and $\left(\alpha_{i}\right)_{1}^{k} \subseteq \mathbb{R}$

$$
F\left(\left(\alpha_{i}\right)_{1}^{k}\right) \equiv \varlimsup_{n_{1} \rightarrow \infty} \ldots \varlimsup_{n_{k} \rightarrow \infty}\left\|\sum_{i=1}^{k} \alpha_{k} z_{n_{i}}\right\|=\left\|\left(\alpha_{i}\right)\right\|_{\infty} .
$$

We prove this by induction on $k$. For $k=1$ the result is obvious. Assume that (16) holds for $k^{\prime}<k$ with $k>1$. From (2.2.5), applied twice,

$$
\begin{aligned}
F\left(\left(\alpha_{i}\right)_{1}^{k}\right) & \leq \max \left\{\left\|\left(\alpha_{i}\right)\right\|_{\infty}, \varlimsup_{n_{1} \rightarrow \infty} \ldots \varlimsup_{n_{k} \rightarrow \infty}\left\|\sum_{i=1}^{k} \alpha_{i} z_{n_{i}}\right\|_{B}\right\} \\
& \leq \max \left\{\left\|\left(\alpha_{i}\right)\right\|_{\infty}, \varlimsup_{n_{1} \rightarrow \infty} \ldots \varlimsup_{n_{k} \rightarrow \infty}\left\|\sum_{i=1}^{k} \alpha_{i} z_{n_{i}}\right\|_{A}\right\} .
\end{aligned}
$$

By (13) we see that there exists $k^{\prime}<\infty$ so that

$$
\varlimsup_{n_{1} \rightarrow \infty} \ldots \varlimsup_{n_{k} \rightarrow \infty}\left\|\sum_{i=1}^{k} \alpha_{i} z_{n_{i}}\right\|_{A}=\varlimsup_{n_{1} \rightarrow \infty} \ldots \varlimsup_{n_{k} \rightarrow \infty}\left\|\sum_{i=1}^{k} \alpha_{i} z_{n_{i}}\right\|_{\bigcup_{\ell=2}^{k^{\prime}} A_{\ell}} .
$$

There exists $2 \leq \ell \leq k^{\prime}$ so that this

$$
=\varlimsup_{n_{1} \rightarrow \infty} \ldots \varlimsup_{n_{k} \rightarrow \infty}\left\|\sum_{i=1}^{k} \alpha_{i} z_{n_{i}}\right\|_{A_{\ell}} .
$$

From Lemma 3.5 this limit is not bigger than

$$
\varlimsup_{n_{1} \rightarrow \infty} \ldots \varlimsup_{n_{k} \rightarrow \infty} \frac{1}{\ell} \sum_{j=1}^{\ell}\left\|\sum_{i=k_{j}-1}^{k_{j}} \alpha_{i} z_{n_{i}}\right\|
$$

for some $1 \leq k_{0} \leq k_{1} \leq \cdots \leq k_{\ell} \leq k$. Thus from the induction hypothesis this is in turn either $\leq\left\|\left(\alpha_{i}\right)\right\|_{\infty}$ if $k_{i} \in(1, k)$ for some $i$ or otherwise,

$$
\leq \frac{\ell-1}{\ell}\left\|\left(\alpha_{i}\right)\right\|_{\infty}+\frac{1}{\ell} F\left(\left(\alpha_{i}\right)_{1}^{k}\right) \text {. }
$$

In the latter case $\frac{\ell-1}{\ell} F\left(\left(\alpha_{i}\right)_{1}^{k}\right) \leq \frac{\ell-1}{\ell}\left\|\left(\alpha_{i}\right)\right\|_{\infty}$ and so we deduce that (16) holds (the upper $\infty$-estimate implies the lower one).

Using (16) we can construct a block basis $\left(\bar{y}_{n}\right)$ of $\left(z_{n}\right)$ of the form $\bar{y}_{n}=$ $\sum_{i=1}^{\bar{k}_{n}} z_{m(n, i)}$ for some $\bar{k}_{n} \uparrow \infty$ and $\bar{k}_{n}<m(n, 1)<\cdots<m\left(k, \bar{k}_{n}\right)$ with $\bar{y}_{n}$ being (essentially) an $\ell_{\infty}^{\bar{k}_{n}}$-average. There is a slight difficulty in that $\left\|z_{n}\right\| \rightarrow 1$ as 
opposed to $\left\|z_{n}\right\|=1$ but we shall ignore this trivial obstacle. We may presume that for some $\varepsilon_{i} \downarrow 0$,

(17) for $i \in \mathbb{N}$ :

a) $\bar{y}_{i}$ is an $\ell_{\infty}^{\bar{k}_{i}}$-average of $\left(z_{n}\right)$ with constant $1+\varepsilon_{i}$.

b) If $\left(x_{1}^{*}, \ldots, x_{j}^{*}\right)$ is $(\tilde{\mathfrak{A}}, M, \sigma)$ or $(\mathfrak{B}, M, \sigma)$-admissible with $\max \operatorname{supp} x_{j}^{*} \geq$ min supp $\bar{y}_{i}$, then

$$
\left|x^{*}\left(\bar{y}_{i}\right)\right|<\varepsilon_{i} \text { for } x^{*} \in \bigcup_{t \geq \sigma\left(x_{1}^{*}, \ldots, x_{j}^{*}\right)} A_{t} \cup \bigcup_{t \geq \sigma\left(x_{1}^{*}, \ldots, x_{j}^{*}\right)} B_{t} .
$$

c) There exist $y_{i}^{*} \in A_{\bar{k}_{i}}$ with

$$
y_{i}^{*}\left(\bar{y}_{i}\right)>\frac{1}{3} \text { and } k_{i+1} \geq \sigma\left(y_{1}^{*}, \ldots, y_{i}^{*}\right) .
$$

Part b) is achieved via (13) and (15). Also (17) c) yields that:

(18) If $M_{m}<n_{1}<\cdots<n_{m}$, then

$$
\left\|\sum_{i=1}^{m} \bar{y}_{m_{i}}\right\| \geq\left\|\sum_{i=1}^{m} \bar{y}_{m_{i}}\right\|_{B_{m}}>\frac{1}{3} \frac{m}{f(m)} .
$$

We can also assume the following growth condition.

$$
\begin{aligned}
& \text { a) } \quad 1-\varepsilon_{1}-\frac{1}{f(2)}>0, \text { and } \\
& \text { b) } \quad \text { for } i \in \mathbb{N}, \quad \varepsilon_{i+1}<\frac{\varepsilon_{i}}{i f^{-1}\left(\frac{i \max \operatorname{supp} \bar{y}_{i}}{\varepsilon_{i}}\right)} .
\end{aligned}
$$

Conditions (17)-(19) yield that conditions (1)-(4) hold for the sequence $\left(\bar{y}_{i}\right)$ replacing $\left(y_{i}\right)$. We shall now show that the sequence $\left(\bar{y}_{i}\right)$ satisfies, for some $C$, that for all $m$ there exists $n_{0}$ so that if $n_{0}<n_{1}<\cdots<n_{m}$, then $\left\|\sum_{1}^{m} \bar{y}_{n_{i}}\right\| \leq C \frac{m}{f(m)}$. Thus we return to Case 1 and the proof will be complete.

Choose $m_{0}$ so that

$$
\begin{aligned}
& \text { a) } \frac{1}{f(2)}+\frac{6 f\left(m_{0}\right)}{m_{0}}<1-\varepsilon_{1} \\
& \text { b) }\left(1+2 \varepsilon_{m_{0}}+\frac{1}{f(2)}\right)\left(1+\varepsilon_{m_{0}}\right)+\varepsilon_{m_{0}}<2 \text {, and } \\
& \text { c) } \frac{m_{0}}{f\left(m_{0}\right)}>3 .
\end{aligned}
$$

Define

$$
C=\max \left\{2 m_{0}, \frac{10}{1-\varepsilon_{1}-\frac{1}{f(2)}}\right\} .
$$

Our claim is trivial for $m \leq m_{0}$. Let $m>m_{0}$. Using (17) b) choose $x=\sum_{1}^{m} x_{i}$ where $x_{i}=\bar{y}_{n_{i}}, m<n_{1}<\cdots<n_{m}$ and where $n_{1}$ is so large that

$$
\left\|\bar{y}_{n}\right\|_{B_{\ell}}<\frac{\varepsilon_{m}}{m^{2}+2}
$$

for all $n, \ell \geq n_{1}$. We first show that for $\ell \geq 2$,

$$
\|x\|_{C_{\ell}}<\|x\|\left(1-\varepsilon_{1}\right) .
$$


Conditions (17) b) and (19) b) imply that Lemma 2.2 a) holds for $\left(\frac{\bar{y}_{n_{i}}}{1+\varepsilon_{m}}\right)_{1}^{m}$ in the setting $\mathcal{D}=\mathfrak{B}, \varepsilon=\varepsilon_{m}$ and $k=n_{1}$. Thus for $\ell \geq 2$, by (2.2.2),

$$
\begin{aligned}
\|x\|_{C_{\ell}} \leq & \frac{1}{f(\ell)}\|x\|_{B}+\frac{\min (\ell, m)}{f(\ell)} \sup _{\ell \geq n_{1}}\|x\|_{B_{\ell}} \\
& \quad+\left(1+\varepsilon_{m}\right)\left(\frac{1}{f(\ell)}+1+2 \varepsilon_{m}\right)+\frac{2}{f(\ell)} \max _{i \leq m} \sup _{\ell \geq n_{1}}\left\|x_{i}\right\|_{B_{\ell}} \\
\leq & \frac{1}{f(\ell)}\|x\|_{B}+\left(1+\varepsilon_{m}\right)\left(\frac{1}{f(\ell)}+1+2 \varepsilon_{m}\right)+\frac{\min (\ell, m) m+2}{f(\ell)} \max _{\substack{i \leq m \\
\ell \geq n_{1}}}\left\|x_{i}\right\|_{B_{\ell}} \\
\leq & \frac{1}{f(\ell)}\|x\|_{B}+\left(1+\varepsilon_{m}\right)\left(\frac{1}{f(\ell)}+1+2 \varepsilon_{m}\right)+\varepsilon_{m} .
\end{aligned}
$$

We used (21) to get the last estimate. By (20) b) this is $\leq \frac{1}{f(\ell)}\|x\|_{B}+2$. Thus by (18),

$$
\begin{aligned}
\|x\|_{C_{\ell}} & \leq \frac{1}{f(\ell)}\|x\|_{B}+6 \frac{f(m)}{m}\|x\|_{B} \\
& \leq\left[\frac{1}{f(\ell)}+\frac{6 f(m)}{m}\right]\|x\| \leq\left(1-\varepsilon_{1}\right)\|x\|
\end{aligned}
$$

by (20) a).

Finally, if $\ell \geq 2$ and if $\|x\|_{B_{\ell}}>\left(1-\varepsilon_{1}\right)\|x\|$, then by (4)

$$
\begin{aligned}
\|x\|_{B_{\ell}} & \leq \frac{\|x\|}{f(\ell)}+3+9 \frac{\min (\ell, m)}{f(\ell)} \\
& \leq \frac{\|x\|}{f(\ell)}+3+9 \frac{m}{f(m)} \\
& \leq \frac{\|x\|}{f(\ell)}+10 \frac{m}{f(m)}
\end{aligned}
$$

(using $3 \leq \frac{m_{0}}{f\left(m_{0}\right)}<\frac{m}{f(m)}$ by $(20)$ c)). Thus

$$
\left(1-\varepsilon_{1}\right)\|x\|-\frac{1}{f(\ell)}\|x\|<\frac{10 m}{f(m)}
$$

and so by our choice of $C$,

$$
\|x\| \leq\left(\frac{10}{1-\varepsilon_{1}-\frac{1}{f(\ell)}}\right) \frac{m}{f(m)} \leq C \frac{m}{f(m)} .
$$

This, thankfully, concludes the proof.

\section{REFERENCES}

[AD] S. Argyros and I. Deliyanni, Examples of asymptotically $\ell^{1}$ Banach spaces, Trans. Amer. Math. Soc. 349 (1997), 973-955. MR 97f:46021

[BHO] S. Bellenot, R. Haydon and E. Odell, Quasi-reflexive and tree spaces constructed in the spirit of R.C. James, Contemporary Math. 85 (1989), 19-43. MR 89m:46014

[CS] P.G. Casazza and T.J. Shura, Tsirel'son's Space, Lectures Notes in Math., vol. 1363, Springer-Verlag, Berlin and New York, 1989. MR 90b:46030

[FJ] T. Figiel and W.B. Johnson, A uniformly convex Banach space which contains no $\ell_{p}$, Compositio Math. 29 (1974), 179-190. [MR 50:8011

[GM] W.T. Gowers and B. Maurey, The unconditional basic sequence problem, J. Amer. Math. Soc. 6 (1993), 851-874. MR 94k:46021 
[J] R.C. James, Uniformly nonsquare Banach spaces, Ann. Math. 80 (1964), 542-550. MR 30:4139

[K] J.L. Krivine, Sous-espaces de dimension finie des espaces de Banach réticulés, Ann. of Math. 104 (1976), 1-29. MR 53:11341

[L] H. Lemberg, Nouvelle démonstration d'un theorem de J.L. Krivine sur la finie representation de $\ell_{p}$ dans un espace de Banach, Israel Journal of Mathematics 39 (1981), 391-398. MR 83b:46015

[MMT] B. Maurey, V.D. Milman and N. Tomczak-Jaegermann, Asymptotic infinite-dimensional theory of Banach spaces, Operator Theory, Advances and Applications 77 (1995), 149175. MR 97g: 46015

[MR] B. Maurey and H. Rosenthal, Normalized weakly null sequences with no unconditional subsequences, Studia Math. 61 (1971), 77-98. MR 55:11010

[OS] E. Odell and Th. Schlumprecht, On the richness of the set of $p$ 's in Krivine's theorem, Operator Theory, Advances and Applications 77 (1995), 177-198. MR 96i:46015

[P] V. Ptak, A combinatorial theorem on systems of inequalities and its application to analysis, Czech. Math. J. 84 (1959), 629-630. MR 22:890

[T] B. S. Tsirelson, Not every Banach space contains $\ell_{p}$ or $c_{0}$, Funct. Anal. Appl. 8 (1974), 138-141.

Department of Mathematics, University of Texas at Austin, Austin, Texas 78712-1082

E-mail address: odell@math.utexas.edu

Department of Mathematics, Texas A \& M University, College Station, Texas 77843

E-mail address: thomas.schlumprecht@math.tamu.edu 